\title{
comportement réel et théorique de quelques ouvrages
}

par

\section{Édouard Recordon}

Professeur de Mécanique des Sols à l'Ecole Polytechnique

Fédérale de Lausanne

\section{COMPORTEMENT REEL. ET THEORIOUE DE QUELQUES OUVRAGES}

La comparaison entre le comportement d'un ouvrage et les prévisions faites à l'époque du projet est l'une des préoccupations majeures des ingénieurs.

L'examen de quelques cas concrets, choisis parmi les problèmes de stabilité de pentes, de déformations sous remblais ou sous plaques chargées et de transferts de chaleur dans une nappe souterraine, permet de mettre en évidence les causes principales des écarts constatés entre les observations et le modèle théorique.

L'objectif est de poser un certain nombre de questions sur l'accent qu'il conviendrait de mettre, selon le problème étudié, sur les prospections, les propriétés des matériaux ou les modèles mathématiques par exemple, pour améliorer les prévisions. C'est aussi de présenter quelques études types exécutées en Suisse française.

\section{ACTUAL AND THEORETICAL BEHAVIOUR OF A FEW WORKS}

The comparison between the behaviour of a work and the forecasts made at the time of the project is one of the major concerns of engineers.

The examination of a few actual cases, chosen among the problems of stability of slopes, of deformations under embankments or under loaded plates and of heat transfers in an underground water table, makes it possible to bring out the main causes of the deviations found between observations and the theoretical model.

The objective is to raise a certain number of questions as to the stress that should be given, depending upon the problem studied, to the prospections, the properties of the materials or the mathematical models for example, in order to improve the forecasts. It is also to present a number of standard studies carried out in French Switzerland. 



\title{
comportement réel et théorique de quelques ouvrages (*)
}

\author{
par Edouard RECORDON
}

\section{INTRODUCTION}

Des études très nombreuses ont eu pour but, ces dix ou vingt dernières années, d'affiner les méthodes de laboratoire permettant de déterminer les propriétés mécaniques des sols et de façonner des outils mathématiques d'une finesse très grande pour l'analyse des phénomènes. Ce faisant, on a peut-être parfois perdu de vue que les causes des écarts existant entre comportement réel et théorique d'un ouvrage sont à rechercher dans la nature, c'est-à-dire dans l'hétérogénéité du matériau, ou dans son contexe géologique. Peut-être est-il illusoire de développer des méthodes d'essais de laboratoire ou des méthodes de calcul très sophistiquées, si par ailleurs les données d'entrée, telles que les conditions hydrologiques ou paramètres des sols, sont incertaines.

Ce type de considération est probablement à l'origine des tentatives, de plus en plus nombreuses actuellement, d'introduire en géotechnique la méthode probabiliste, en considérant les caractéristiques des sols et les diverses sollicitations des ouvrages comme des variables aléatoires.

Les études présentées dans les congrès internationaux sont plus le reflet, nous semble-t-il, des recherches qui se font dans les universités et dans les bureaux d'étude que celui des observations faites sur les chantiers par les constructeurs. Il est extrêmement difficile de réunir des observations et des résultats de mesures permettant la comparaison entre le comportement réel et théorique d'un ouvrage. On constate souvent, après coup, que l'explication du comportement réel aurait nécessité d'autres mesures que celles qui ont été faites. Nous nous sommes donc fixé un but difficile à atteindre. Nous tenterons, néanmoins, à l'aide de quelques exemples, de mettre en évidence quelques-une des principales causes des différences existant entre les phénomènes réels observés et leur prévision théorique. Ces exemples sont tirés, pour la plupart, d'études exécutées, ces dernières années, par le laboratoire de géotechnique de 1'Ecole Polytechnique Fédérale de Lausanne.

Le terme d'« ouvrage » que nous avons utilisé dans le titre, par raison de commodité, n'est peut-être pas exactement celui que nous aurions souhaité trouver. II doit être pris ici dans un sens large car il recouvre, en fait, l'objet étudié ; il s'agira de pentes naturelles ou de talus, de nappes souterraines, de remblais ou de plaques chargées.

\section{GLISSEMENT DE LA CORNALLAZ}

\subsection{Conditions générales}

Le premier cas concret que nous examinons est celui de la stabilité d'une pente naturelle qui a posé un problème difficile dans le cadre de la construction de l'autoroute Lausanne-Simplon (fig. 1) (**). Cette autoroute doit relier la Suisse française à l'Italie du Nord. Le glissement de la Cornallaz $\mathrm{ABC}$ est la partie amont, audessus de la ligne de chemin de fer, d'un phénomène d'instabilité qui, de l'altitude $700 \mathrm{~m}$ environ, s'étend jusqu'au lac dont le niveau est à l'altitude 370 . La partie inférieure $\mathrm{CD}$ est le glissement des Luges.

Dans la première partie $A B$ du glissement de la Cornallaz, la direction du mouvement est peu différente de celle du pendage des couches de molasse et la pente de $13^{\circ}$ n'est que légèrement inférieure à celle de la molasse. Dans le secteur BC, la direction du mouvement forme un angle de $45^{\circ}$ environ avec la direction du pendage et la pente du terrain est beaucoup plus faible. Au droit du point $C$, se trouve le remblai du chemin de fer, qui existe depuis une centaine d'années et n'a guère bougé. La partie $\mathrm{CD}$ du phénomène est donc séparée de la

(*) Conférence présentée devant le Comité français de Mécanique des Sols, le 16 mai 1977.

$\left({ }^{\star *}\right)$ Figure tirée de l'étude de M. le Professeur A. Bersier, géologue. partie supérieure. Dans cette zone inférieure, les masses glissées ne se déplacent plus sur le toit des couches de molasse, le coteau naturel a été formé lors de l'érosion de la cuvette du lac Léman; le plan du coteau coupe donc les couches de molasse en tranches. Ce secteur ne fait pas l'objet de notre étude. Nous ne nous occuperons que de la partie $\mathrm{ABC}$ qui domine l'autoroute et la ligne de chemin de fer.

Ces glissements se trouvent dans une région où les couches de molasse sont inclinées de 15 à $20^{\circ}$ dans une direction sensiblement parallèle à la rive du lac. La molasse est une roche sédimentaire mais sa composition est très variable (fig. 2). Certaines couches sont constituées d'une argile très compacte, d'autres de grès sableux plus ou moins grossier. Le grès est beaucoup plus dur et résistant que les couches argileuses. Lorsque les argiles sont mises au contact de l'air, l'humidité, les variations de température et le gel les attaquent fortement. L'altération des couches argileuses étant plus rapides que celle des couches de grès, ces dernières restent en relief. Le plan de rupture de la Cornallaz se situe dans l'une des couches argileuses. La rupture qui s'est produite a permis l'infiltration des eaux dans une fissure très fine que l'on a pu observer et suivre, dans cette couche d'argile, tout au long d'une galerie de drainage, exécutée pour stabiliser le mouvement. 
Fig. 1, - Plan de situation des glissements de la Cornallaz.

AR : Autoroute; CFF : Chemin de fer.

$\mathrm{ABC}$ : Glissement de la Cornallaz; CD Glissement des Luges.

I : Zone d'alimentation par érosion de la molasse.

2 : Zone des glissements.
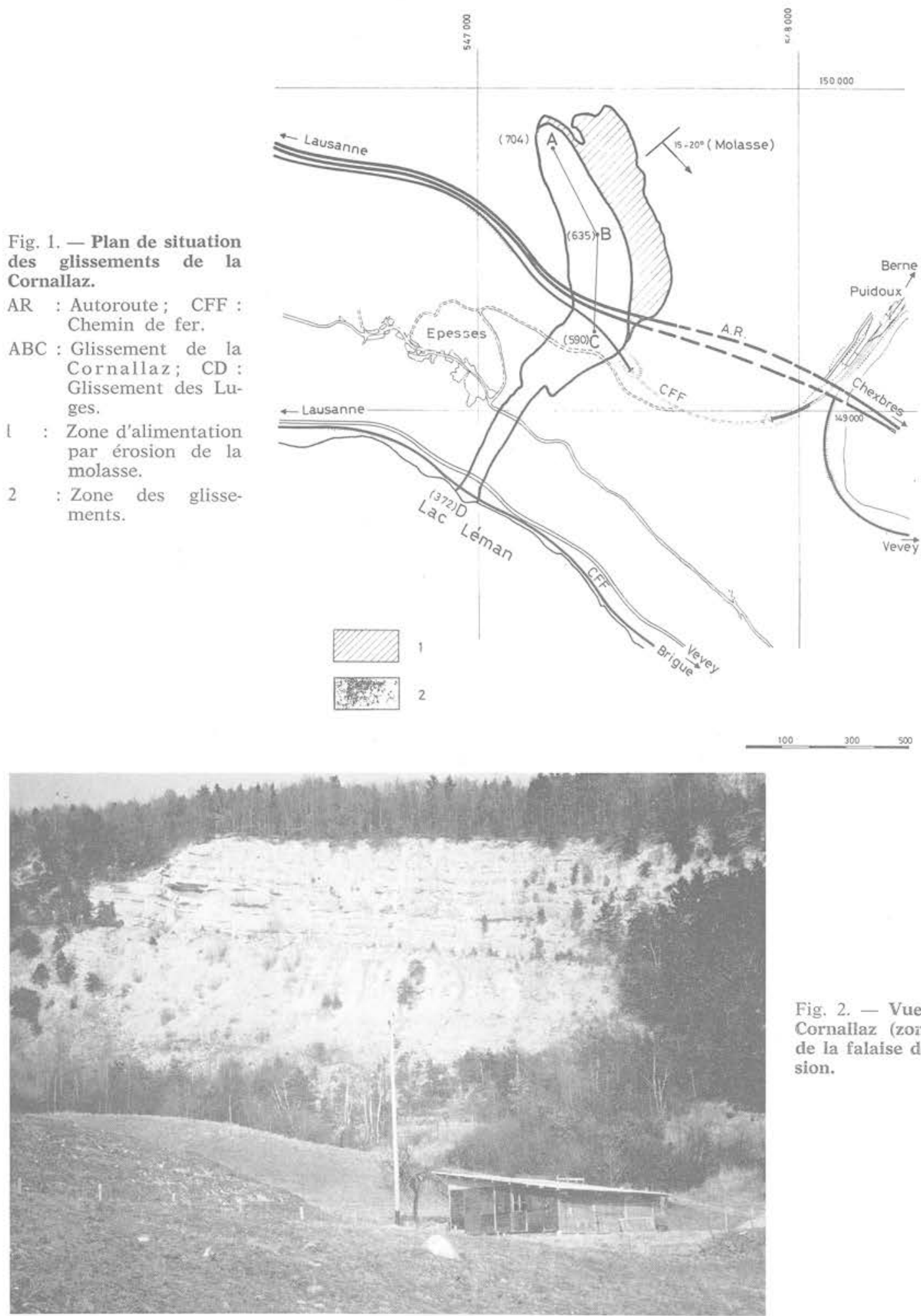

Fig. 2. - Vue du glissement de la Cornallaz (zone des points BC) et de la falaise de molasse, zone d'érosion.

Ce glissement très ancien est étudié depuis la fin du siècle dernier, époque de construction de la ligne de chemin de fer Lausanne-Berne qui passe au-dessous. Il est périodiquement actif. La dernière fois, en 1950 environ, un déplacement des masses de 10 à $20 \mathrm{~m}$ se produisit et les terres vinrent s'appuyer contre le remblai de chemin de fer, La circulation des trains dut être interrompue pendant plusieurs semaines. On peut expliquer que le remblai soit resté stable pendant de nombreuses années par l'effet des galeries de drainage construites auparavant et par les conditions topographiques.

La construction de l'autoroute qui franchit ce glissement a nécessité, il y a une dizaine d'années, une étude très détaillée : les deux facteurs géologiques à l'origine de ce mouvement de pente sont d'une part, l'érosion de la falaise de molasse, niche du décrochement primitif, dont les matériaux tombent et viennent charger graduellement la partie amont des couches instables (fig. 2) et d'autre part, l'existence. à certaines époques, dans une 
Fig. 3. - Modèle physique du glissement de la Cornallaz.

1. Surface de rupture dans la molasse.

2. Ligne d'énergie $=$ niveau piézométrique (théorique).

3. Glissement des Luges.

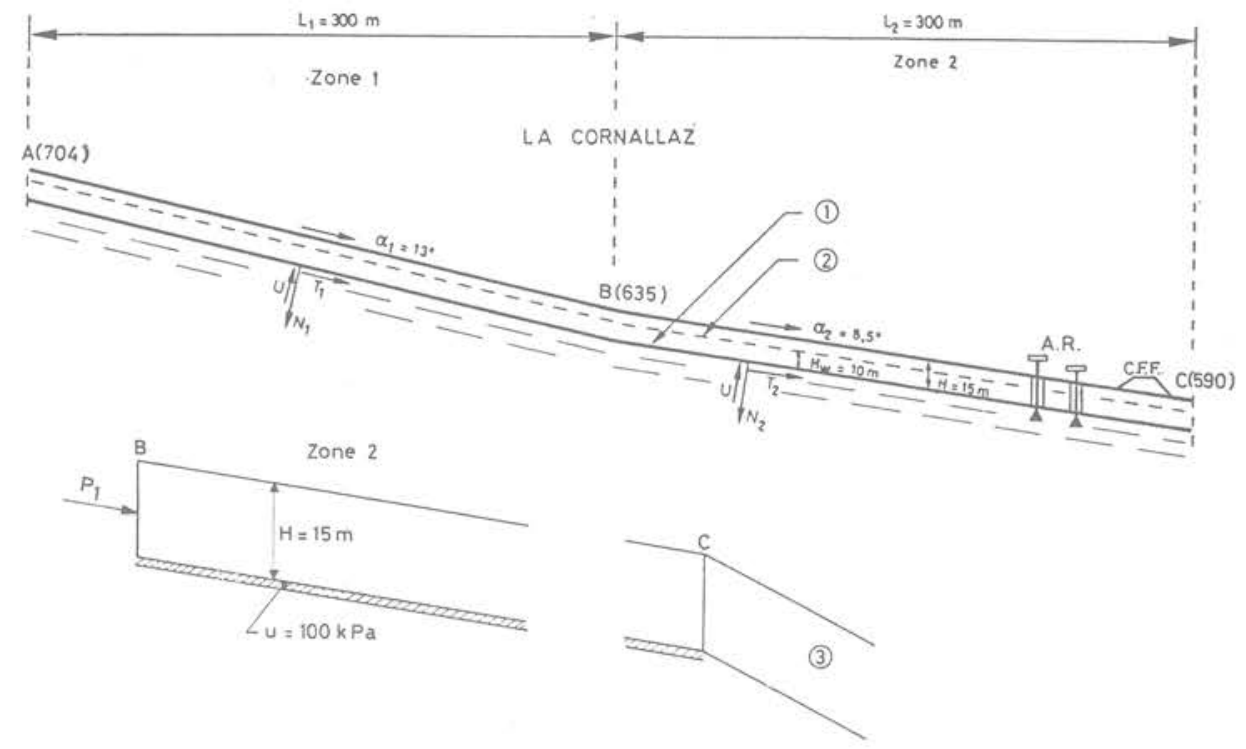

couche située à $15 \mathrm{~m}$ de profondeur environ, de pressions interstitielles importantes, de l'ordre de $100 \mathrm{kPa}$ (1 bar).

\subsection{Le modèle de calcul}

La figure 3 représente le modèle physique du glissement de la Cornallaz. La géométrie du glissement est beaucoup plus compliquée que ne le montre cette figure. La zone $1(\mathrm{AB})$ forme, en plan, un angle $\beta=25^{\circ}$ avec la zone $2(\mathrm{BC})$. Les directions de $\mathrm{AB}$ et de $\mathrm{BC}$ n'étant pas celles du pendage de la molasse, les angles $\alpha_{1}$ et $\alpha_{2}$, inclinaison de la surface de glissement sur l'horizontale, sont plus petits que ce pendage de $20^{\circ}$ (voir fig. 1).

Le modèle mathématique très simplifié que nous adoptons pour mettre en évidence les phénomènes principaux est celui qui traduit l'équilibre de la zone 2 (BC). Le facteur de sécurité s'écrit :

$$
\mathrm{F}_{\mathrm{BC}}=\frac{\left(\mathrm{N}_{2}-\mathrm{U}_{2}\right) \operatorname{tg} \Phi^{\prime}}{\mathrm{T}_{2}+\mathrm{P}_{1}}
$$

La poussée $P_{1}$ qu'exerce la zone 1 sur la zone 2 est donnée par :

$$
\begin{aligned}
& \mathrm{P}_{1}=\left[\mathrm{T}_{1}-\left(\mathrm{N}_{1}-\mathrm{U}_{1}\right) \operatorname{tg} \Phi^{\prime}\right] \cos \left(\alpha_{1}-\alpha_{2}\right) \cos \beta \\
& \beta=25^{\circ}: \text { angle en plan entre } \mathrm{AB} \text { et BC. } \\
& \gamma=20 \mathrm{kN} / \mathrm{m}^{3} \quad \mathrm{H}=15 \mathrm{~m} \quad \mathrm{~L}_{1}=300 \mathrm{~m} \\
& \gamma_{w} \simeq 10 \mathrm{kN} / \mathrm{m}^{3} \quad \mathrm{H}_{w}=10 \mathrm{~m} \quad \mathrm{~L}_{2}=300 \mathrm{~m}
\end{aligned}
$$$$
\gamma=20 \mathrm{kN} / \mathrm{m}^{3} \quad \mathrm{H}=15 \mathrm{~m} \quad \mathrm{~L}_{1}=300 \mathrm{~m} \quad \alpha_{1}=13^{\circ} \Phi^{\prime}=16^{\circ}
$$$$
\alpha_{2}=8.5^{\circ} c=0
$$

Les mouvements du glissement se sont toujours produits en périodes de pluie. C'est donc que la résistance est influencée par les pressions interstitielles et qu'il s'agit de résistance par frottement et non par cohésion. $\mathrm{La}$ valeur maximum $\Phi=16^{\circ}$ correspond à un facteur de sécurité de 1 . Il s'agit évidemment d'un angle de frottement résiduel. Des forages de reconnaissance ont montré que les pressions interstitielles existaient réellement à certaines époques, l'eau remontant d'une dizaine de mètres dans les forages dès qu'ils atteignaient le plan de rupture.

\subsection{Dispositions constructives}

Dans ce cas, les premiers travaux ont consisté à creuser une galerie de captage des eaux dans l'axe du glissement (fig. 4) $\left(^{*}\right)$. Son rôle est d'assurer un drainage parfait et d'empêcher la remontée des pressions interstitielles. De plus, les appuis du pont ont été reportés sur la molasse (fig. 4) et les piles des ponts traversent la couche instable à l'intérieur de puits de protection

(*) Figure tirée de la publication [2].

Si $u=100 \mathrm{kPa}$ (pression sur la surface de rupture)

$$
\begin{aligned}
& \mathrm{N}_{1}=\gamma \mathrm{HL}_{1} \cos \alpha_{1}=87693 \mathrm{kN} / \mathrm{m} \quad \mathrm{N}_{2}=\gamma \mathrm{H} \mathrm{L}_{2} \cos \alpha_{2}=89011 \mathrm{kN} / \mathrm{m} \\
& \mathrm{U}_{1}=\gamma_{w} \mathrm{H}_{w} \mathrm{~L}_{1} / \cos \alpha_{1}=30789 \mathrm{kN} / \mathrm{m} \quad \mathrm{U}_{2}=\gamma_{w} \mathrm{H}_{w} \mathrm{~L}_{2} / \cos \alpha_{2}=30333 \mathrm{kN} / \mathrm{m} \\
& \left(\mathrm{N}_{1}-\mathrm{U}_{1}\right) \operatorname{tg} \Phi^{\prime}=16317 \mathrm{kN} / \mathrm{m} \quad\left(\mathrm{N}_{2}-\mathrm{U}_{2}\right) \operatorname{tg} \Phi^{\prime}=16826 \mathrm{kN} / \mathrm{m} \\
& \mathrm{T}_{1}=\gamma \mathrm{HL}_{1} \sin \alpha_{1}=20246 \mathrm{kN} / \mathrm{m} \quad \mathrm{T}_{2}=\gamma \mathrm{H} \mathrm{L} \sin \alpha_{2}=13303 \mathrm{kN} / \mathrm{m} \\
& \mathrm{P}=3550 \mathrm{kN} / \mathrm{m} \\
& F_{B C}=\frac{16826}{13303+3550}=1.00 \\
& \text { Si } u=0 \\
& \mathrm{~N}_{1}=87693 \mathrm{kN} / \mathrm{m} \quad \mathrm{N}_{2}=89011 \mathrm{kN} / \mathrm{m} \\
& \mathrm{U}_{1}=0 \quad \mathrm{U}_{2}=0 \\
& \left(\mathrm{~N}_{1}-\mathrm{U}_{1}\right) \operatorname{tg} \Phi^{\prime}=25184 \mathrm{kN} / \mathrm{m} \quad\left(\mathrm{N}_{2}-\mathrm{U}_{2}\right) \operatorname{tg} \Phi^{\prime}=25523 \mathrm{kN} / \mathrm{m} \\
& \mathrm{T}_{1}=20246 \mathrm{kN} / \mathrm{m} \quad \mathrm{T}_{2}=13303 \mathrm{kN} / \mathrm{m} \\
& P_{1}=0 \operatorname{car}\left(N_{1}-U_{1}\right) \operatorname{tg} \Phi^{\prime}>T_{1} \\
& \mathrm{~F}_{\mathrm{BC}}=\frac{25}{13303+0}=1.92
\end{aligned}
$$




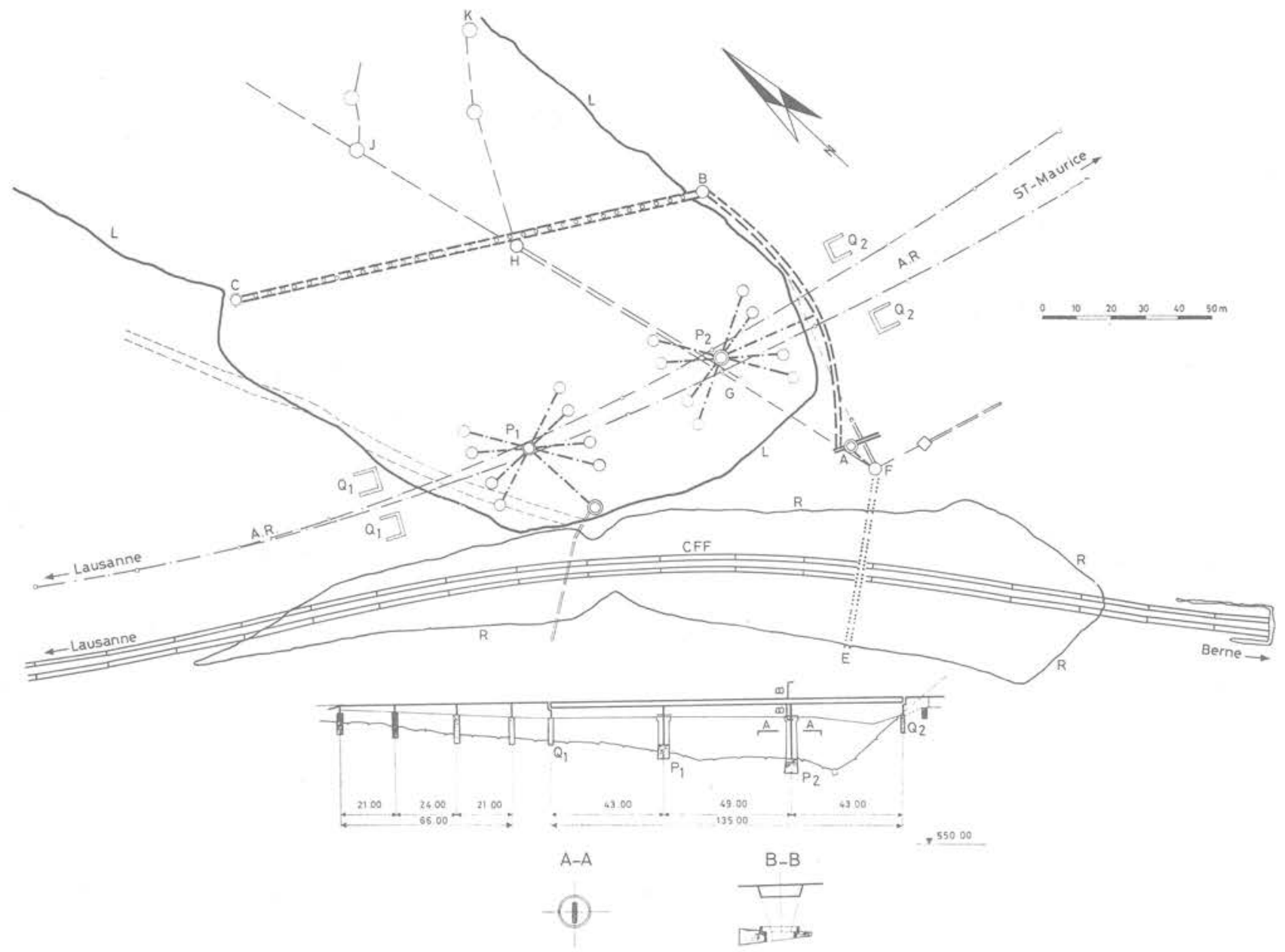

Fig. 4. - Plan des drainages et profil en long du pont de l'autoroute.

AR : Autoroute; CFF : Chemin de fer; R : limite du remblai CFF.

$\mathrm{L}$ : Limites du glissement de $1950: \mathrm{P}_{1} \mathrm{P}_{2}$ : appui des ponts dans puits $\varnothing 5 \mathrm{~m}$ avec drains rayonnants; $\mathrm{Q}_{1} \mathrm{Q}_{2}$ culées des ponts; EFGH : Galerie de drainage ancienne, visitable; HJK : Galerie bloquée ancienne; AB : Nouvelle galerie visitable; BC : Nouvelle galene drainante avec drains verticaux $\varnothing 50 \mathrm{~cm}$, tous les $4 \mathrm{~m}$.

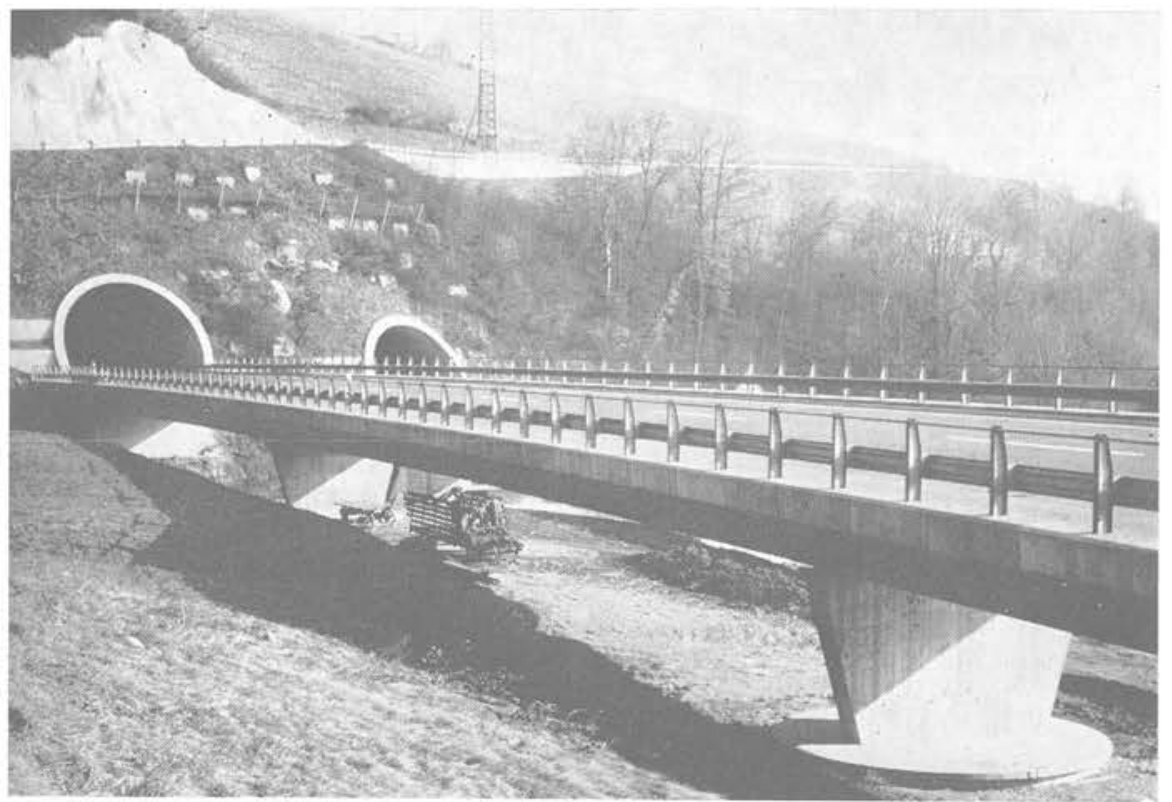

Fig. 5. - Vue du pont de l'autoroute et d'un puits entourant une palée du pont.

de $5 \mathrm{~m}$ de diamètre laissant un vide minimal de $1 \mathrm{~m}$ environ entre leur paroi et la pile du pont. Ce vide doit permettre, en cas de catastrophe, un certain mouvement du puits avant qu'il ne touche les piles (fig. 5).

\subsection{Conditions hydrauliques}

Il est intéressant d'insister ici sur le rôle des pressions hydrauliques dans l'étude de stabilité. Prenons l'exemple simple d'une tranche reposant sur un plan de glissement parallèle à la surface du terrain et inclinée de 


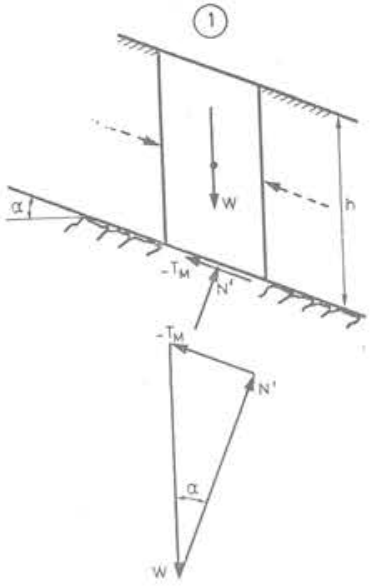

(2)

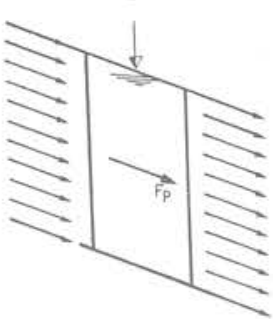

(3)
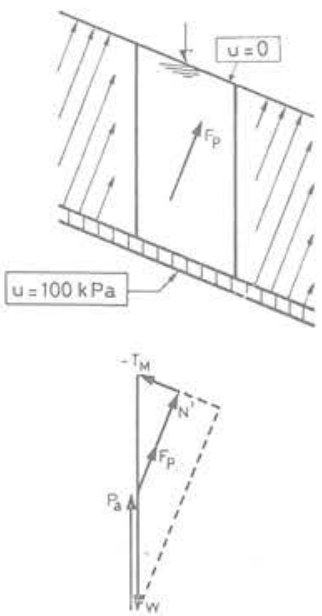

Fig. 6. - Conditions hydrauliques possibles et polygones des forces, selon l'hypothèse de Fellenius.

$\alpha=20^{\circ}$. La figure 6 représente cette couche et les dynamiques des forces dans l'hypothèse de Fellenius; les forces latérales s'annulent. Pour diverses conditions hydrauliques, le facteur de sécurité est égal à :

$$
\mathrm{F}=\frac{\mathrm{N}^{\prime} \operatorname{tg} \Phi^{\prime}}{\mathrm{T}_{\mathrm{M}}}
$$

si l'on admet que le sol n'a pas de cohésion.

En choisissant les valeurs numériques suivantes : $h=8 \mathrm{~m}$, largeur horizontale de la tranche $b=4 \mathrm{~m}$, $\gamma=20 \mathrm{kN} / \mathrm{m}^{3}, \mathrm{~W}=640 \mathrm{kN}, \Phi^{\prime}=30^{\circ}$, on est conduit aux valeurs suivantes du facteur de sécurité :
Dans le tableau ci-dessous, on a :

$\mathrm{P}_{a}=$ Poussée d'Archimède $\quad \mathrm{F}_{p}=$ Force de percolation $\mathrm{U}=u \frac{b}{\cos \alpha}$

Cas $2 \mathrm{~F}_{p}=\gamma_{w} \mathrm{~V} \sin \alpha=109 \mathrm{kN}$

Cas $3 \quad \mathrm{~F}_{p}=\gamma_{w} \mathrm{~V} \frac{10-h \cos ^{2} \alpha}{h \cos \alpha}=125 \mathrm{kN}$

Le dernier cas est de beaucoup le plus défavorable et correspond à la mise en pression d'une fissure dont le toit est suffisamment imperméable pour que, pendant la durée d'augmentation des pressions, sur le plan de glissement, l'eau n'ait pas le temps de pénétrer dans la masse. Ces conditions sont plus défavorables en particulier que celles du cas 3 qui pourtant est très semblable : la pression sur le plan de rupture est la même dans les deux cas, donc la résistance au cisaillement est la même; par contre, dans le cas 3 la masse instable est déjaugée, alors qu'elle ne l'est pas dans le cas 4. La force motrice du mouvement est donc plus grande dans 4 que dans 3 .

Le glissement de la Cornallaz, que nous avons décrit plus haut, correspond, comme l'ont montré les prospections et les travaux de puits et galeries, au cas 4 que nous venons de citer.

\subsection{Géologie}

Les conditions géologiques de la Suisse romande et du site de la Cornallaz sont étroitement liées à celles de la formation des Alpes. Comment expliquer, en effet, la forte inclinaison, de l'ordre de 15 à $20^{\circ}$, des couches de molasse alors que partout ailleurs, sur le Plateau suisse, ces couches sédimentaires sont sensiblement horizontales? Cette explication ne peut être donnée sans un bref aperçu de l'origine géologique de ces matériaux.

La carte géologique de la Suisse montre quatre grandes régions géologiques :

. le Plateau suisse, formé de ce que nous appelons la molasse et que nous décrirons de manière détaillée plus loin ;

- le Jura, constitué de couches calcaires à plis relativement réguliers, fortement karstifiés ;

- les Hautes-Alpes, granitiques au sud de la vallée du Rhône et calcaires au nord de cette même vallée;

- les Préalpes formées principalement de calcaires et de flysch.

A la fin du XIX $X^{c}$ siècle et au début de celui-ci, les géologues ont élaboré la théorie de l'orogenèse des Alpes et du Jura. Ces phénomènes de soulèvement très lents, dont la vitesse devait être de l'ordre de $1 \mathrm{~cm} /$ année, ont eu lieu au cours de l'ère tertiaire et

\begin{tabular}{|c|c|c|c|}
\hline $\begin{array}{c}\text { Cas } \\
\mathrm{n}^{\circ}\end{array}$ & $\mathrm{N}^{\prime} \operatorname{tg} \Phi^{\prime}$ & $\mathrm{T}_{\mathrm{M}}$ & $\mathrm{F}$ \\
\hline 1 & $\mathrm{~W} \cos \alpha \operatorname{tg} \Phi^{\prime}=347 \mathrm{kN}$ & $\mathrm{W} \sin \alpha=219 \mathrm{kN}$ & 1.58 \\
\hline 2 & $\left(\mathrm{~W}-\mathrm{P}_{a}\right) \cos \alpha \operatorname{tg} \Phi^{\prime}=174 \mathrm{kN}$ & $\left(\mathrm{W}-\mathrm{P}_{a}\right) \sin \alpha+\mathrm{F}_{p}=219 \mathrm{kN}$ & 0.79 \\
\hline 3 & {$\left[\left(\mathrm{~W}-\mathrm{P}_{a}\right) \cos \alpha-\mathrm{F}_{p}\right] \operatorname{tg} \Phi^{\prime}=101 \mathrm{kN}$} & $\left(\mathrm{W}-\mathrm{P}_{a}\right) \sin \alpha=109 \mathrm{kN}$ & 0.93 \\
\hline 4 & $(\mathrm{~W} \cos \alpha-\mathrm{U}) \operatorname{tg} \Phi^{\prime}=101 \mathrm{kN}$ & $\mathrm{W} \sin \alpha=219 \mathrm{kN}$ & 0.46 \\
\hline
\end{tabular}


ont duré vraisemblablement plusieurs dizaines de millions d'années. Remarquons qu'une vitesse moyenne de $1 \mathrm{~cm} /$ année correspond à un soulèvement de $10000 \mathrm{~m}$ en un million d'années. Il n'est pas déraisonnable de penser que les Alpes ont atteint, à l'ère tertiaire, une altitude beaucoup plus grande que celle qu'elles ont aujourd'hui. L'arc alpin est dû à une poussée tectonique horizontale, tangentielle à la croûte terrestre, partant de la Méditerranée et dirigée vers le nord, due peut-être à un mouvement de tout le continent africain.

Les alpes en se soulevant, de même que le Jura d'ailleurs, ont émergé des mers dans lesquelles s'étaient sédimentés, à l'ère secondaire, les calcaires du Jura et des Alpes (fig. 7) $\left.{ }^{*}\right)$. La mer refoulée vers le nord a été, à certaines époques, coupée par le Jura qui délimitait un bassin fermé au-dessus du Plateau suisse. La
1) Le long de la plaine du Rhône, à l'amont du lac Léman, on retrouve la molasse sous les couches des Préalpes sur une vingtaine de kilomètres de longueur.

2) En bordure des Alpes, les couches de la molasse sont fortement inclinées vers les Alpes alors que sur le Plateau elles sont sensiblement horizontales. Ces deux régions sont délimitées par une grande faille que l'on peut suivre depuis la Savoie jusqu'en Suisse alémanique; elle est sensiblement parallèle au pied des Préalpes.

Le site de la Cornallaz se trouve à $5 \mathrm{~km}$ environ au sud-est de la faille et fait partie de la région de la molasse inclinée. Les couches gréseuses et argileuses s'y succèdent en bancs de quelques décimètres d'épaisseur, fortement fissurés du fait des mouvements qu'ils ont subis.

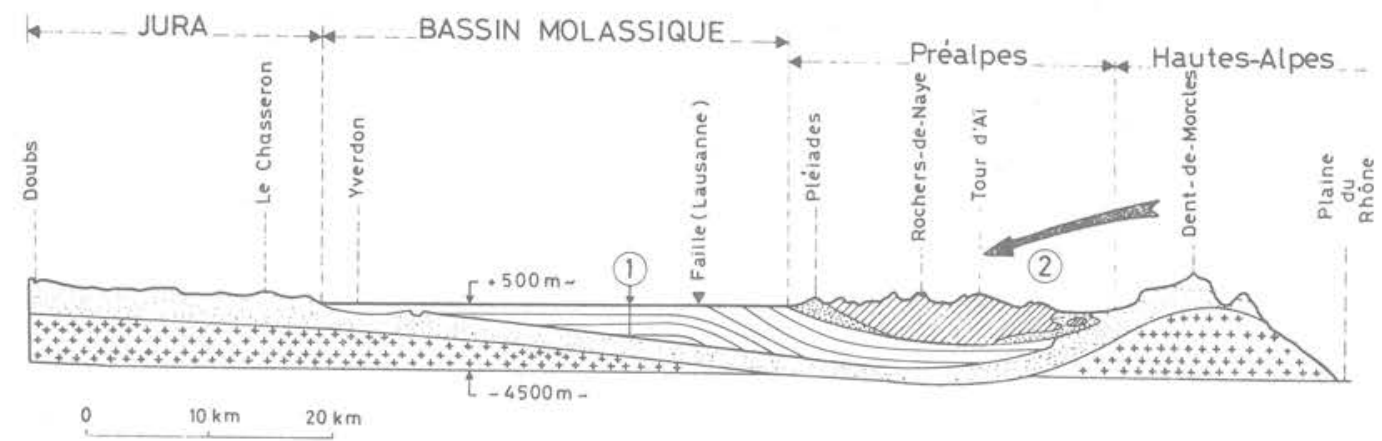

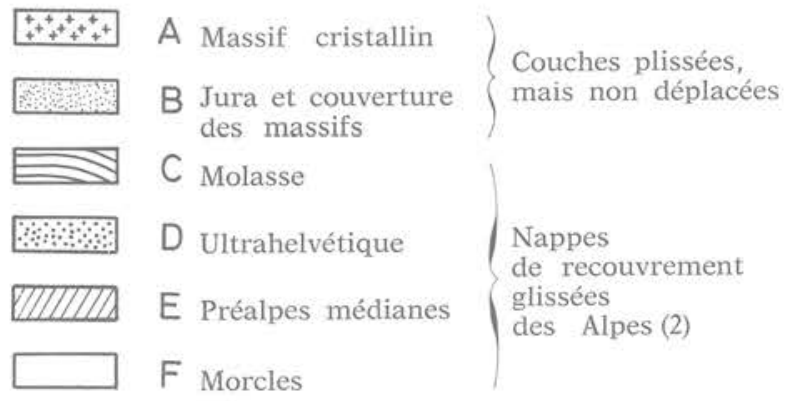

(1) Faille dans la molasse longeant le pied des Préalpes.

Fig. 7. - Coupe géologique de la Suisse française du nord-ouest au sud-est.

nature de la molasse du Plateau démontre que cette roche tendre s'est formée par sédimentation des matériaux d'érosion, transportés par les rivières descendant des Alpes dans des nappes d'eau parfois salées, donc reliées à la mer, et douces à d'autres époques. Elle est constituée d'argile très fortement consolidée, désignée souvent par les entrepreneurs sous le nom de marne, et de grès à matrice limoneuse ou sableuse. En règle générale, les bancs gréseux sont plus abondants du côté des Alpes et les argiles plus fines prédominent du côté du Jura ce qui étaye la thèse de la provenance alpine des matériaux. L'épaisseur la plus grande des couches molassiques est de l'ordre de 3000 à $4000 \mathrm{~m}$.

Deux observations essentielles ont conduit à la théorie des nappes de recouvrement qui explique la géologie des Préalpes. Selon cette théorie, les couches superficielles de flysch et de roches calcaires ont glissé, ou plutôt flué par nappes successives, du haut des Alpes vers le nord et ont recouvert en partie la molasse du Plateau. Ces deux observations sont les suivantes :

(*) Figure tirée de la publication [4].
Les caractéristiques mécaniques de ces matériaux sont très variables. Leurs valeurs se situent entre les extrêmes suivants :

Résistance à la compression simple : $q_{u}=1$ à 10 $\mathrm{MPa}$ (10 à 100 bars).

Modules d'élasticité : $\mathrm{E}=1000$ à $10000 \mathrm{MPa}\left(10^{4}\right.$ à $10^{5}$ bars),

Les valeurs les plus faibles correspondent aux bancs argileux et les plus fortes aux bancs gréseux.

Cette parenthèse géologique nous a paru utile pour situer les conditions de la Suisse et pour montrer que dans notre pays, comme dans toute région de montagne, la connaissance de l'histoire géologique est importante dans l'explication de certains phénomènes géotechniques. Contrairement aux grands pays de plaine qui nous entourent, nous sommes souvent confrontés à des problèmes où mécanique des roches et mécanique des sols interfèrent; de plus, l'action répétée de l'érosion glaciaire est à l'origine de formations très variées et hétérogènes.

\subsection{Prévision des phénomènes}

Il nous a paru intéressant de présenter le problème de la Cornallaz en premier lieu; c'est probablement dans l'étude de la stabilité de pentes naturelles que l'écart entre les phénomènes réels et les prévisions est le plus grand. Une très grande incertitude pèse sur les conditions hydrauliques les plus défavorables. En ce qui concerne les paramètres de résistance des matériaux, il nous parait difficile, dans un tel cas, de les déterminer en laboratoire. Il faudrait disposer d'une boîte de cisaillement de dimensions suffisantes pour permettre de très grands déplacements et permettant en outre d'introduire une pression hydraulique dans la fissure de rupture.

Il était plus simple et plus sûr dans le cas présent, puisqu'il y avait eu rupture, de calculer ces paramètres de résistance par une étude de stabilité avec coefficient 
de sécurité égal à 1 , ce qui nous a donné une valeur globale moyenne de la résistance.

Notons un fait curieux que nous n'expliquons pas : la valeur de l'angle de frottement interne effectif résiduel des argiles compactes de la molasse ne dépasse pas 15 à $16^{\circ}$, même pour une cohésion nulle et des conditions hydrauliques très défavorables. Cette valeur est sensiblement plus basse que celle que l'on trouve généralement dans les manuels et nous l'avons retrouvée dans l'étude de plusieurs glissements.

Dans un tel cas, les facteurs essentiels qui sont à l'origine des erreurs d'évaluation sont les conditions hydrauliques les plus défavorables qui sont très diffi- ciles à évaluer : elles varient dans le temps et les piézomètres donnent des valeurs souvent douteuses. C'est pourquoi l'apport du géologue et les observations sur place lors des prospections sont beaucoup plus importantes que les calculs. Il serait, à notre avis, erroné de consacrer de grands efforts à affiner le modèle mathématique. La modification des hypothèses hydrauliques peut modifier le facteur de sécurité de l'ordre de $50 \%$, alors que l'amélioration de la connaissance de la géométrie du plan de glissement et l'utilisation de méthodes de calcul permettant une discrétisation très fine ne conduira probablement qu'à une diminution de l'écart des prévisions de l'ordre de 10 à $20 \%$.

\section{PASSAGE INFERIEUR DE BUSSIGNY}

La construction d'un passage sous la ligne de chemin de fer Lausanne-Neuchâtel pour une route de faible importance conduisit à entailler assez fortement le sol, formé de couches argileuses, comme le montre la figure $8\left({ }^{*}\right)$. Nous ne sommes plus ici dans la molasse, mais dans les couches d'alluvions fines qui la recouvrent et qui se sont déposées dans l'un des sillons creusés par les glaciers près de Lausanne. Ces alluvions postglaciaires sont constituées de limons argileux ou d'argiles limoneuses normalement consolidées, à forte teneur en eau et faible compacité et à très faible résistance au cisaillement. Ce sont par ailleurs des sols peu perméables, La construction de l'ouvrage et celle de la route débutèrent en 1969 et furent achevées en novembre 1970. Quinze jours après la mise en circulation de la route, un léger soulèvement s'étant manifesté, une première correction de la surface de la route fut nécessaire. Puis la route continua à se soulever et atteignit une hauteur de $+1.40 \mathrm{~m}$ par rapport à son niveau de projet, à l'amont du pont. En été 1971, le phénomène se stabilisa et la route fut maintenue en circulation mais avec une vitesse très limitée $(20 \mathrm{~km} / \mathrm{h})$ car pour passer sur la bosse puis sous le pont, une pente de $15 \%$ était nécessaire.

Les études géotechniques entreprises après l'accident par J.-C. Ott, ingénieur-conseil à Genève, montrèrent (fig. 9) que l'on avait dès la surface et jusqu'à une dizaine de mètres de profondeur, une couche d'argile plastique très peu perméable et, entre 10 et $20 \mathrm{~m}$ de profondeur, une couche d'argile molle avec intercalations de varves limoneuses. Les varves sont des couches très fines de moins de $1 \mathrm{~mm}$ d'épaisseur se succédant à quelques $\mathrm{mm}$ ou quelques $\mathrm{cm}$ de distance. Elles correspondent à des cycles saisonniers lors de la sédimentation des alluvions. Les varves, beaucoup plus perméables que les couches d'argile qu'elles séparent, permettent la circulation lente de l'eau et sa mise en pression. Chaque varve de limon peut donc être une couche artésienne.

Lors de l'étude géotechnique préliminaire, les forages à rotation n'avaient pas permis de constater l'existence d'une nappe souterraine mais seulement d'observer quelques très faibles venues d'eau. Après l'accident, la pose de cellules de pression permit de mesurer des pressions très importantes, le niveau piézométrique étant largement au-dessus du terrain (cotes 410 à $412 \mathrm{~m}$ ).

La présence de la nappe artésienne étant démontrée, il fut décidé d'abaisser les pressions dans cette nappe en construisant une lignée de six puits filtrants d'environ

(*) Les figures $8,9,10$, sont tirées de la publication [3] de J.-C. Ott et J. Marguerat.

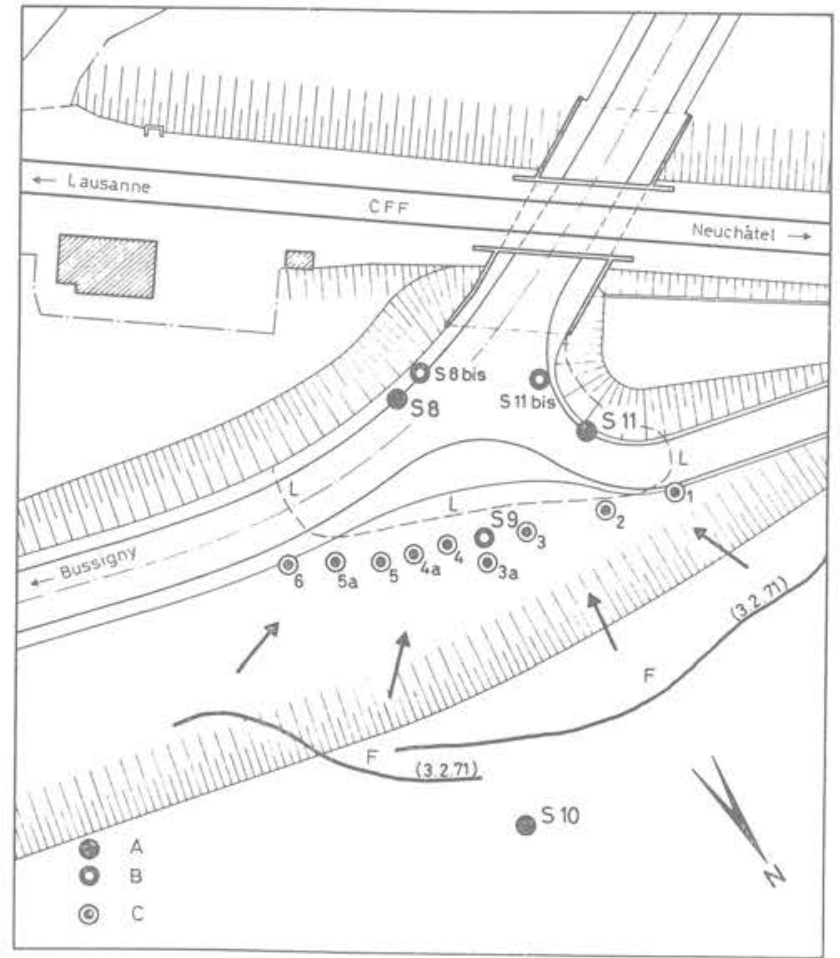

Fig. 8, - Plan du passage inférieur de Bussigny.

A : Sondages avec piézomètres; B: Sondage; C : Puits filtrants; F : Fissures dans le terrain; L : Limite du soulèvement de la route.

$20 \mathrm{~m}$ de longueur, de petit diamètre $(42 \mathrm{~mm}$ intérieur avec filtre de sable de 1 à $2 \mathrm{~mm}$ ). Ces puits permirent d'abaisser les niveaux piézométriques de 4 à $5 \mathrm{~m}$ en une année (voir fig. 9). Néanmoins, la durabilité de l'effet de ces puits ne pouvant pas être garantie à longue échéance, il fut décidé de construire sur toute la zone amont de la route un radier ancré (fig. 10) en profondeur, permettant d'exercer un effort vertical compensant en partie le poids des terres excavées pour la construction de la tranchée de la route.

Les calculs de stabilité effectués par le bureau Ott en introduisant un angle de frottement interne de $8^{\circ}$ et une cohésion variant de $30 \mathrm{kPa}(0.3 \mathrm{bar})$ jusqu'à $5 \mathrm{~m}$ de profondeur à $10 \mathrm{kPa}(0.1 \mathrm{bar})$ entre 7 et $15 \mathrm{~m}$, ont conduit aux facteurs de sécurité suivants :

a) Avant accident et avant construction de

la tranchée de la route, avec sous pressions.

b) Après excavation et sous pressions légèrement diminuées par puits.
$\mathrm{F}=2.48$

$\mathrm{F}=1.12$ 


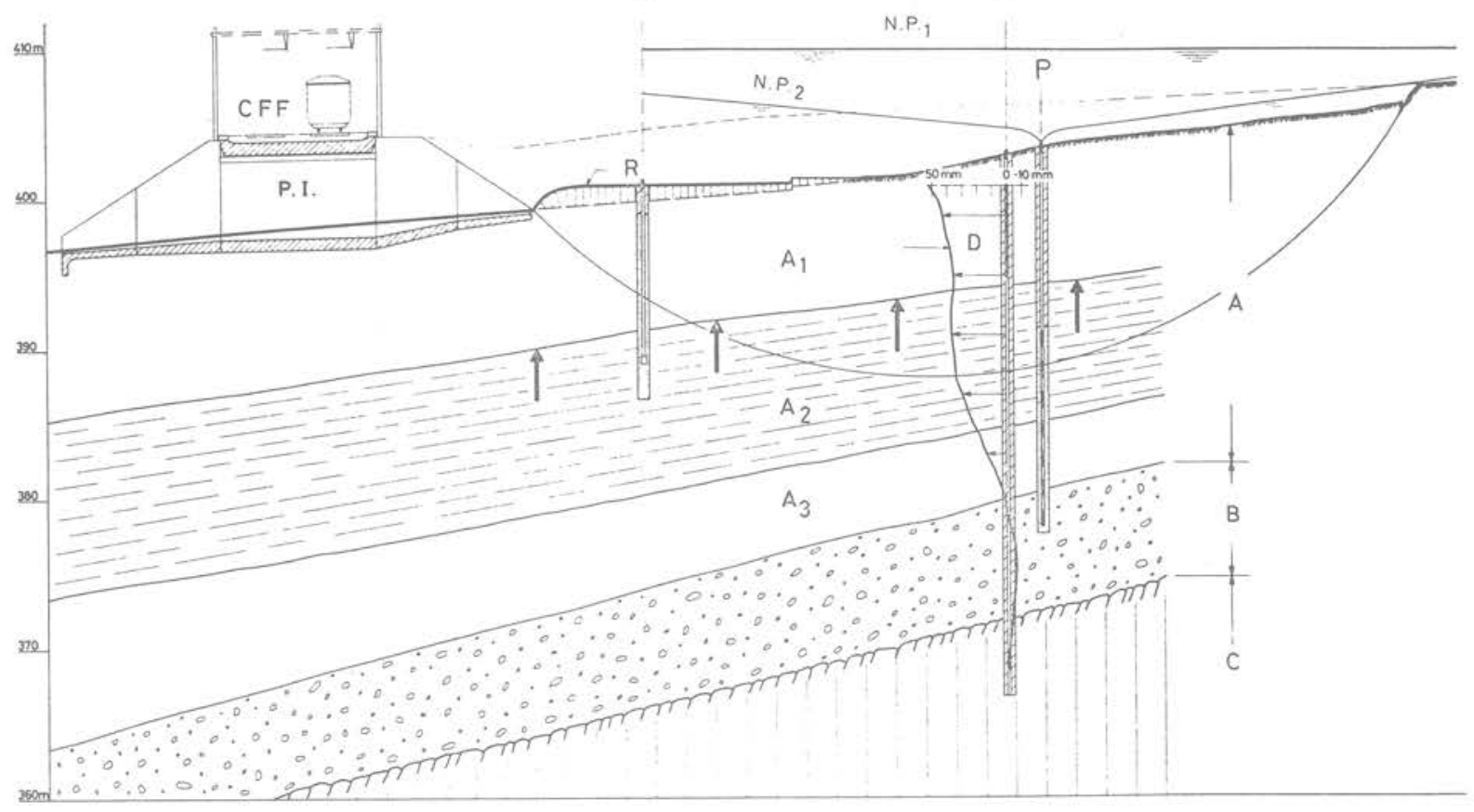
$30 \mathrm{~m}$

Fig. 9. - Modèle physique du glissement de Bussigny.

P.I : Passage inférieur; $\mathrm{R}$ : Profil de la route après glissement; P : Puits filtrants ; D : Déplacements horizontaux; $\mathrm{NP}_{1}$ et $\mathrm{NP}_{2}$ : Niveaux piézométriques avant et après rabattement par puits ; $\mathrm{A}$ : Dépôts glaciolacustres; $A_{1}$ : Argile plastique très peu perméable; $A_{2}$ : Argile molle varvée, couche artésienne; $A_{3}$ : Argile limoneuse ; B : Moraine; C : Molasse.

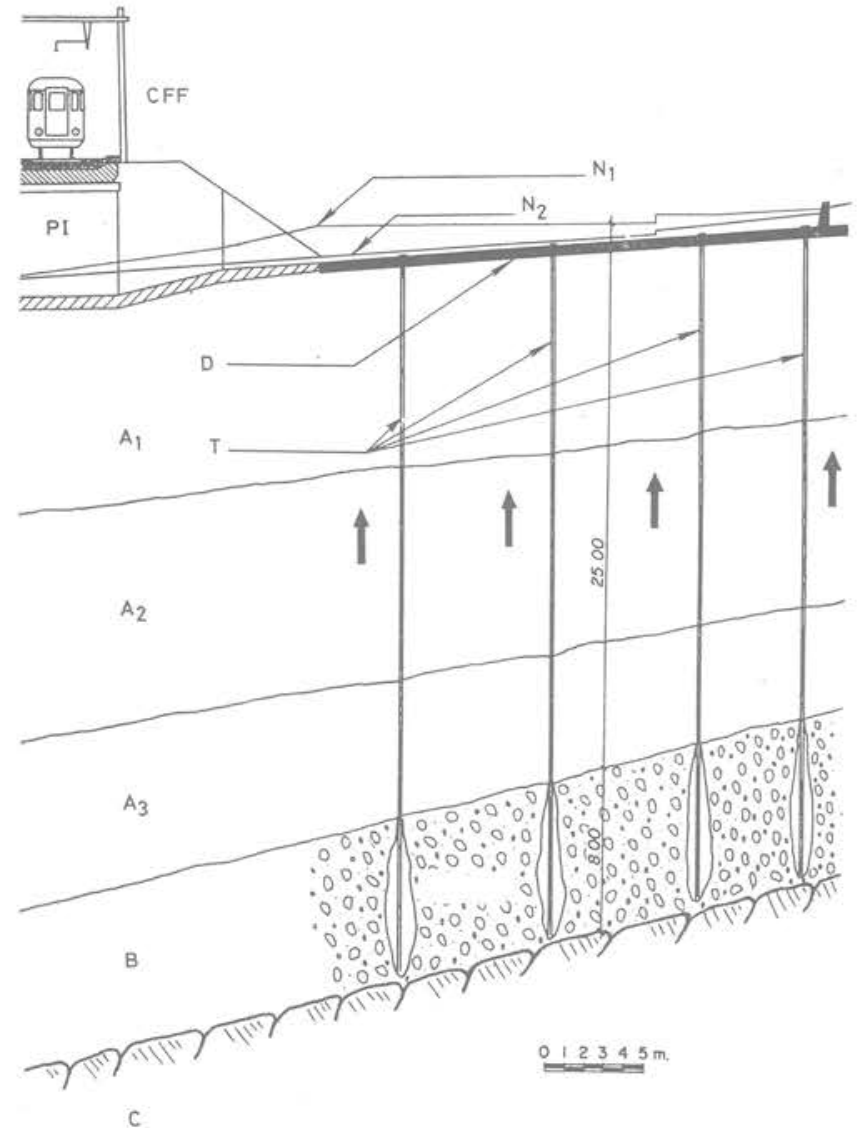

Fig. 10. - Profil du glissement avec radier ancré.

$\mathrm{D}$ : Dalle de béton (radier) en éléments préfabriqués; $\mathrm{T}$ : Ancrages, $\mathrm{N}_{1}$ : Niveau route déformée, $\mathrm{N}_{2}$ : niveau route projetée $A_{1}, A_{2}, A_{3}, B C$, voir fig. 9 . c) Après excavation et sous pressions abaissées jusqu'au niveau du sol.

$\mathrm{F}=1.37$

d) Etat final avec sous pressions abaissées et surcharge de $40 \mathrm{kPa}(0.4$ bar $)$ dans l'emprise de la route.

La figure 11 montre l'aspect des travaux qui furent achevés en juillet 1973. Tous les travaux ont été exécutés en cinq mois. Depuis trois ans, la route, rendue à la circulation, s'est bien comportée.

Dans ce cas, les puits de drainage ne permettant pas de supprimer complètement les pressions interstitielles, ce sont les ancrages qui ont permis d'obtenir une forte augmentation de la sécurité au glissement et de rétablir à peu près l'équilibre primitif.

Cet exemple, original par la solution trouvée pour la stabilisation du glissement, met à nouveau en évidence l'importance des conditions hydrauliques dont la méconnaissance a été la cause principale de l'accident.

Concluons, en ce qui concerne les problèmes de stabilité de pentes, que c'est la catégorie de problèmes géotechniques où les prévisions sont les plus difficiles. L'expérience faite au Massachussetts Institute of Technology en 1974 le confirme. Lors de cette expérience, il avait été demandé à dix experts avertis de dire, après étude détaillée, pour quelle hauteur de surélévation un remblai existant se romprait. Le résultat des prévisions est donné sur la figure 12 (*) par les surfaces hachurées. Après que les experts aient remis leur rapport, un symposium fut organisé auquel participèrent vingt-six auditeurs. Les experts exposèrent les bases de leur estimation, puis les auditeurs donnèrent leur propre évaluation. Elle correspond aux surfaces non hachurées de la figure 12 .

(*) Figure tirée de la publication [4]. 
Fig. 11. - Vue du chantier, construction du radier.

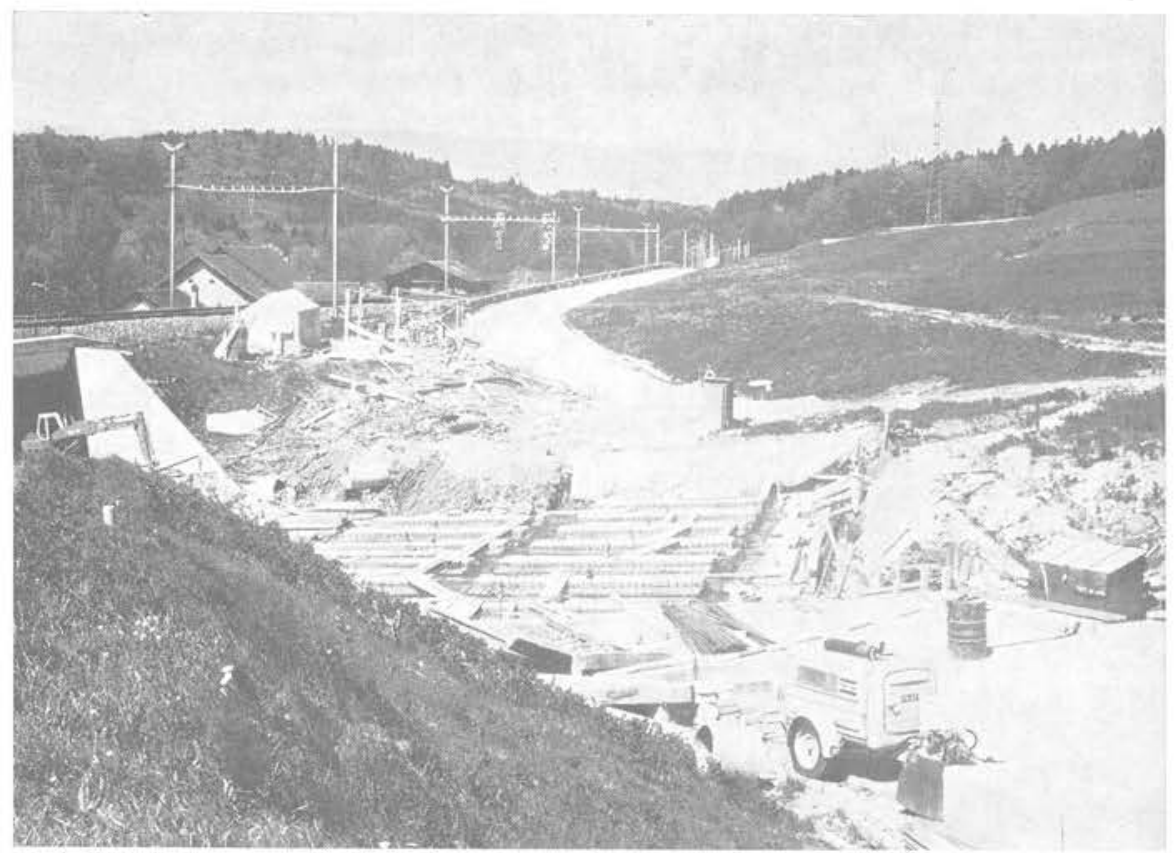

Le résultat est déconcertant, car si l'on avait demandé aux experts d'établir le projet du remblai surélevé de manière à ce qu'il soit stable, ils l'auraient fait correctement. Cela signifie que les erreurs d'évaluation auraient été couvertes par les facteurs de sécurité.

Fig. 12. - Résultats de l'enquête du Massachussetts Institute of Technology, 1973.

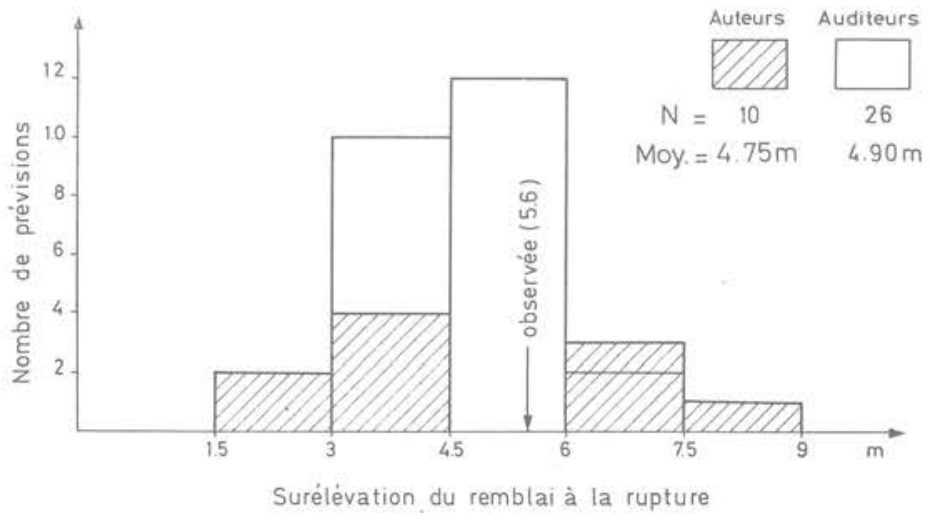

\section{COMPORTEMENT THERMIQUE ET HYDRAULIQUE DE LA NAPPE DU HARD}

(Bâle, voir publication [5])

\subsection{Le site}

La nappe du Hard (fig. 13) se situe sur la rive gauche du Rhin à l'amont de Bâle ; elle est exploitée pour l'alimentation en eau potable de toute la région bâloise dont elle est le principal réservoir. Vingt-et-un millions de $\mathrm{m}^{3}$ d'eau y sont pompés annuellement. Le sous-sol est de plus utilisé comme un immense filtre d'environ $2 \mathrm{~km}$ de longueur et $500 \mathrm{~m}$ de largeur. La nappe est réalimentée par l'eau du Rhin. La figure 13 montre la prise

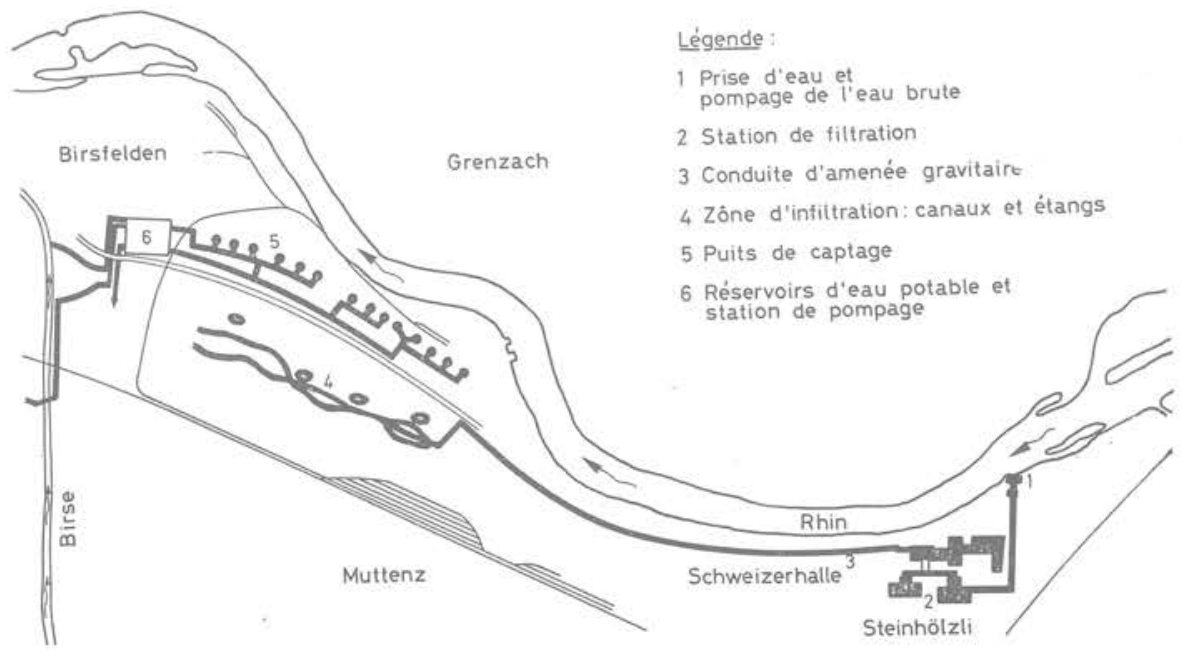

Fig. 13. - Situation des dispositifs de réalimentation et de pompage de la nappe du Hard, à Bâle. 
d'eau dans le Rhin (1) d'où l'eau est pompée dans une station de filtration primaire rapide (2). Elle est ensuite amenée dans la zone d'infiltration (4) située dans la forêt du Hard où l'eau s'infiltre dans le sol à partir de canaux à ciel ouvert et de petits étangs. Une série de puits de captage (5) est installée entre le Rhin et la zone d'infiltration à 300 à $400 \mathrm{~m}$ de cette dernière. L'eau, filtrée durant sa percolation d'environ un mois dans le sol, est conduite au réservoir d'eau potable (6) et à la station de pompage qui alimente le réseau de la ville.

Le débit de réalimentation par infiltrations est environ deux fois plus grand que le débit pompé (40 millions de $\mathrm{m}^{3}$ par an) ce qui assure toujours un écoulement de la nappe vers le Rhin au-delà des puits de captage, de manière à éviter toute pollution.

\subsection{But de l'étude}

La nappe du Hard a été choisie pour tester un modèle mathématique utilisant la méthode des éléments finis et permettant de calculer à toute époque les caractéristiques des flux hydrauliques et thermiques dans une nappe souterraine. Un groupe de travail interdisciplinaire, constitué en 1972 et groupant des chercheurs ingénieurs de deux Instituts de l'Ecole Polytechnique de Lausanne et des géologues du Centre d'Hydrogéologie de l'Université de Neuchâtel $\left({ }^{*}\right)$ a mis sur pied un programme de recherche visant à créer un outil pour l'étude de la pollution thermique et chimique des nappes et pour l'étude du stockage d'énergie sous forme de chaleur. La nappe du Hard présentait l'avantage d'être relativement bien connue dans sa géométrie et du point de vue hydrogéologique. De plus, des mesures de température et de niveau d'eau périodiques avaient été faites dans une série de piézomètres durant toute l'année 1973, ce qui devait permettre de vérifier les calculs de températures et de caler le modèle sur le plan des coefficients de perméabilité.

\subsection{Bases de la simulation}

Une première simulation a été faite par modèle à éléments finis à deux dimensions dans le plan (fig. 14), la troisième dimension étant introduite indirectement par pondération des coefficients hydrauliques et thermiques au droit de chaque nœud.

Deux calculs ont été faits, l'un avec un réseau de 58 éléments et un deuxième en divisant chacun des éléments en quatre parties pour obtenir un réseau de 232 éléments. Comme de coutume, les nœuds du réseau coïncident, autant que faire se peut, avec les puits de captage ou piézomètres existants. Pour le débit de pompage, la densité des puits étant importante, ceux-ci sont traduits par une densité de flux le long des arêtes des éléments en tenant compte de l'épaisseur de l'aquifère.

Dans la zone d'alimentation ou d'injection (zone hachurée de la figure 14), des éléments de taille réduite reproduisent les étangs et l'équivalent des canaux. Le débit y est fixé par unité de surface et par éléments (voir fig. 15).

L'infiltration due aux eaux de pluie étant très faible vis-à-vis du débit de réalimentation artificielle, et par ailleurs les débits pompés et injectés étant sensiblement constants dans le temps, il a été possible dans ce cas d'admettre un régime hydraulique permanent et de n'étudier le régime transitoire que pour le flux thermique.

Les perméabilités introduites sont comprises entre deux valeurs extrêmes $10^{-1} \mathrm{~m} / \mathrm{s}$ et $0.2410^{-2} \mathrm{~m} / \mathrm{s}$ et correspondent aux deux principaux types de sols rencontrés. Un très long travail de calage a permis d'aboutir aux courbes d'isoperméabilité de la figure 15 en procédant par corrections successives de manière à retrou-

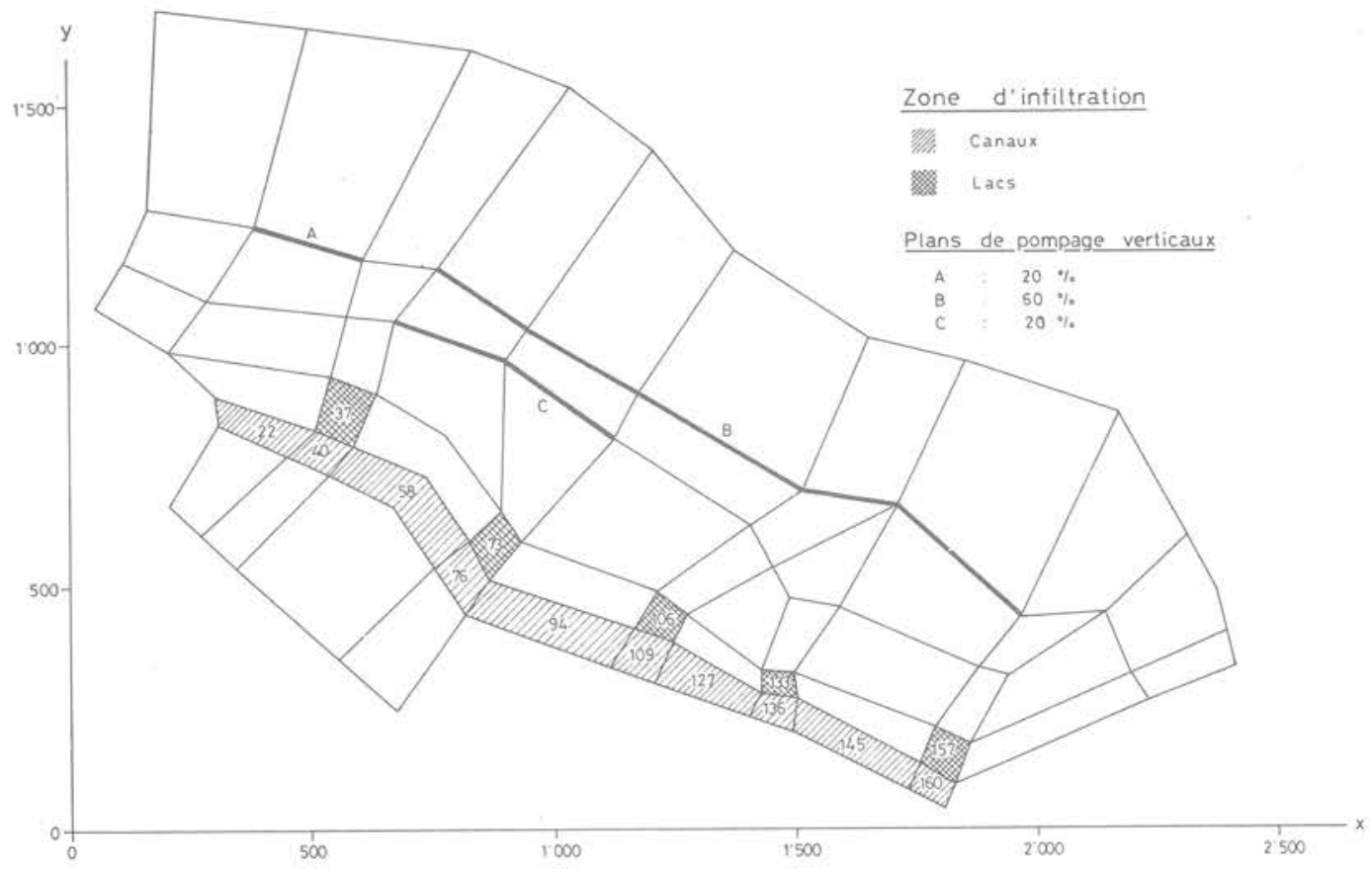

(*) Institut de production d'énergie de l'EPFL, prof. A. Gardel. Centre d'hydrogéologie de l'Université de Neuchâtel, prof. A. Burger. Laboratoire de géotechnique de l'EPFL, prof. E. Recordon.
Fig. 14. - Modèle de calcul du Hard : les cinquante-deux éléments de la nappe à deux dimensions. 


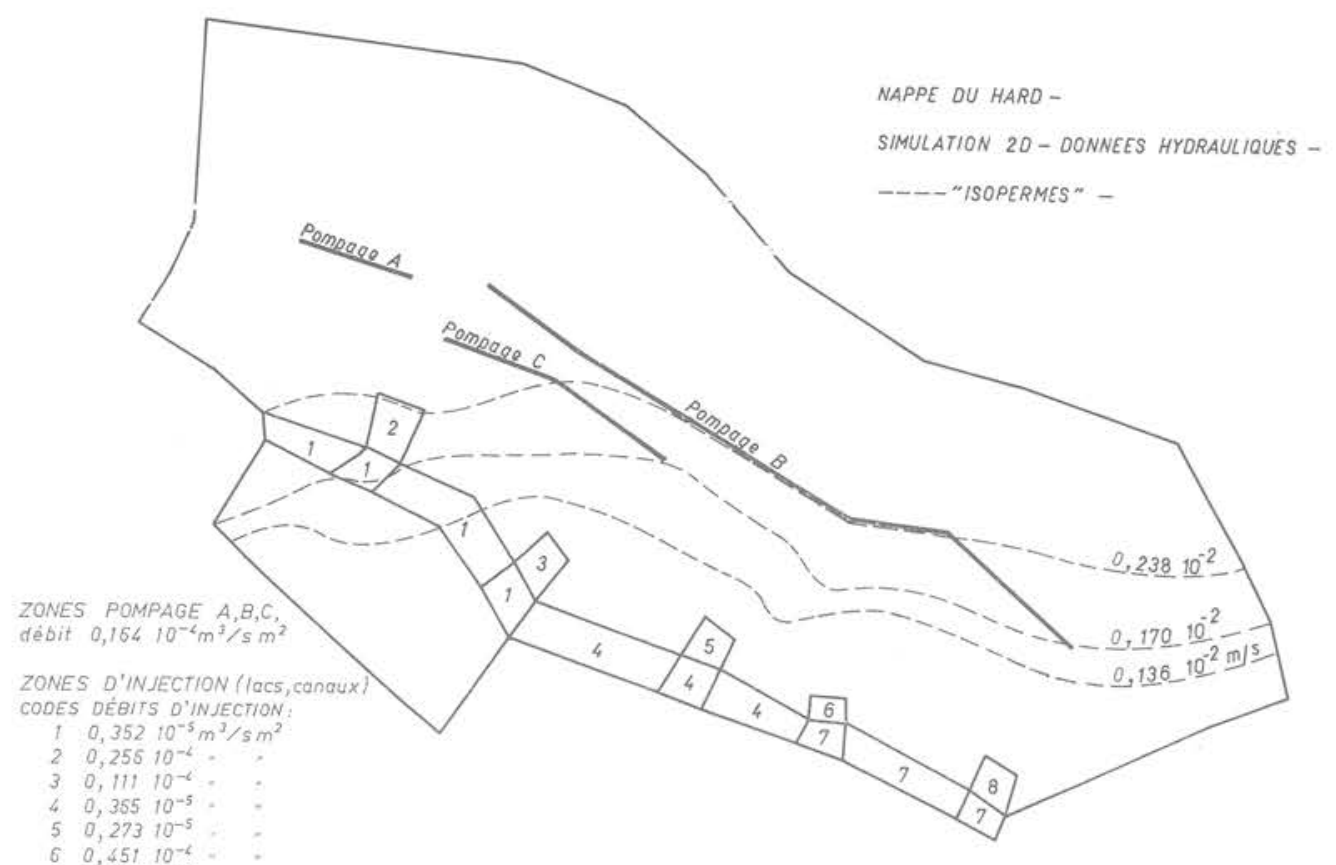

Fig. 15, - Nappe du Hard, courbes d'égale perméabilité,

ver les niveaux mesurés dans les piézomètres. Les conditions hydrauliques limites adoptées sont : le long du Rhin, potentiel imposé et constant au cours du temps. Dans la zone d'injection, la limite extérieure est admise imperméable; le potentiel y est alors une inconnue qui dépend du débit d'apport et de la perméabilité. Dans d'autres zones du contour, on a fixé des conditions du même type compte tenu des indications géologiques. Les surfaces supérieure et inférieure de la nappe sont admises imperméables.

Du point de vue thermique, le réseau utilisé est le même. La conductibilité thermique $\mathrm{K}_{f}=2.5 \mathrm{~W} / \mathrm{mK}$ $\left(0.6 \mathrm{cal} / \mathrm{ms}^{\circ} \mathrm{C}\right)$ et la capacité calorifique $\mathrm{C}=2.32$ $10^{6} \mathrm{~J} / \mathrm{m}^{3} \mathrm{~K}\left(0.555 .10^{6} \mathrm{cal} / \mathrm{m}^{3}{ }^{\circ} \mathrm{C}\right)$ sont admises constantes dans tout le réseau. Le pas de temps est de un mois. La seule condition à la limite imposée est la température de l'eau injectée. Elle est variable et déterminée par un échantillonnage de la courbe des températures de l'eau du Rhin.

Pour simplifier, la température initiale de la nappe a été supposée égale à $10^{\circ} \mathrm{C}$, et le calcul a été fait sur une période de trois cycles annuels, soit trente-six itérations de un mois pour assurer un régime périodique stable.

Une deuxième étude a été entreprise par simulation à trois dimensions, en gardant en plan les mêmes éléments, au nombre de 58 , mais en subdivisant la nappe dans son épaisseur en trois couches. Les données d'input et les conditions aux limites sont restées les mêmes.

\subsection{Résultats des calculs}

La figure 16 montre le résultat du calcul du champ de vitesses au niveau supérieur de la nappe. La direction des vecteurs vitesses permet de voir quelles sont les limites admises comme perméables et celles qui ne le sont pas.

Un autre type de résultats est donné sous forme de cartes des isothermes à diverses époques. La figure 17 correspond au mois de janvier, neuf mois après le début du calcul ( $9^{c}$ itération) et la figure 18 au mois de juillet suivant (15 itération). Au mois de janvier, les températures sont basses dans la zone d'injection et relativements hautes au voisinage des pompages; en été, l'inverse se produit.

Enfin, la figure 19 permet de comparer les valeurs des températures moyennes calculées et mesurées dans quatre piézomètres. La simulation paraît assez bonne puisque les écarts sont en général de 1 à $2^{\circ} \mathrm{C}$ et de $4{ }^{\circ} \mathrm{C}$ au maximum dans les pointes. On remarque que les extremums des courbes calculées sont en général plus marqués que ceux des courbes qui joindraient les points des mesures. Cela peut s'expliquer par une condition limite qui n'a pas été mentionnée jusqu'ici et qui consiste à admettre que le flux thermique est nul à la surface de la nappe.

Cette étude de simulation qui s'est étendue sur deux années de travail est due à M. Menjoz, physicien, et J.D. Favrod, ingénieur civil. Elle a pu être réalisée grâce à la compréhension de la Société Hardwasser AG et avec l'aide de M. Casati dont la connaissance de la nappe du Hard a grandement facilité la compréhension de son comportement [5].

\subsection{Principales causes des écarts entre calculs et mesures}

Les erreurs sur la conductivité et la chaleur spécifique du milieu poreux n'ont pas une grande influence sur les températures calculées. Le flux thermique influence peu les températures ; c'est au contraire le flux hydraulique, auquel correspond le déplacement de masses d'eau avec leur propre température, qui a la plus grande influence. Par ailleurs, la capacité calorifique d'un milieu poreux aquifère varie très peu d'un point à l'autre et une erreur sur la conductivité de l'ordre de $20 \%$ n'a qu'une influence très faible sur les températures calculées. 


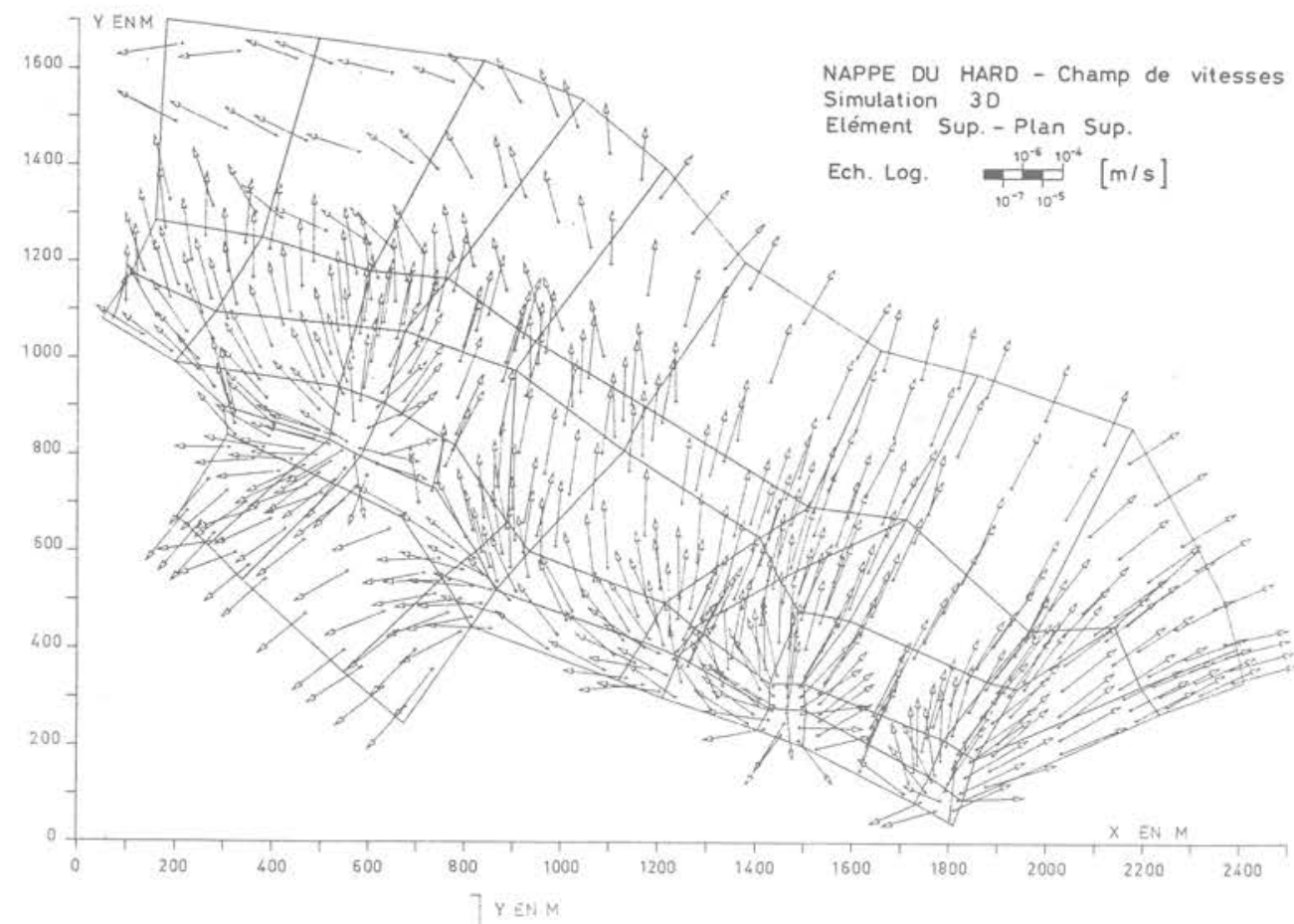

Fig. 16. - Nappe du Hard, champ des vitesses calcule (exemple).

Fig. 17. - Nappe du Hard, isothermes de janvier.

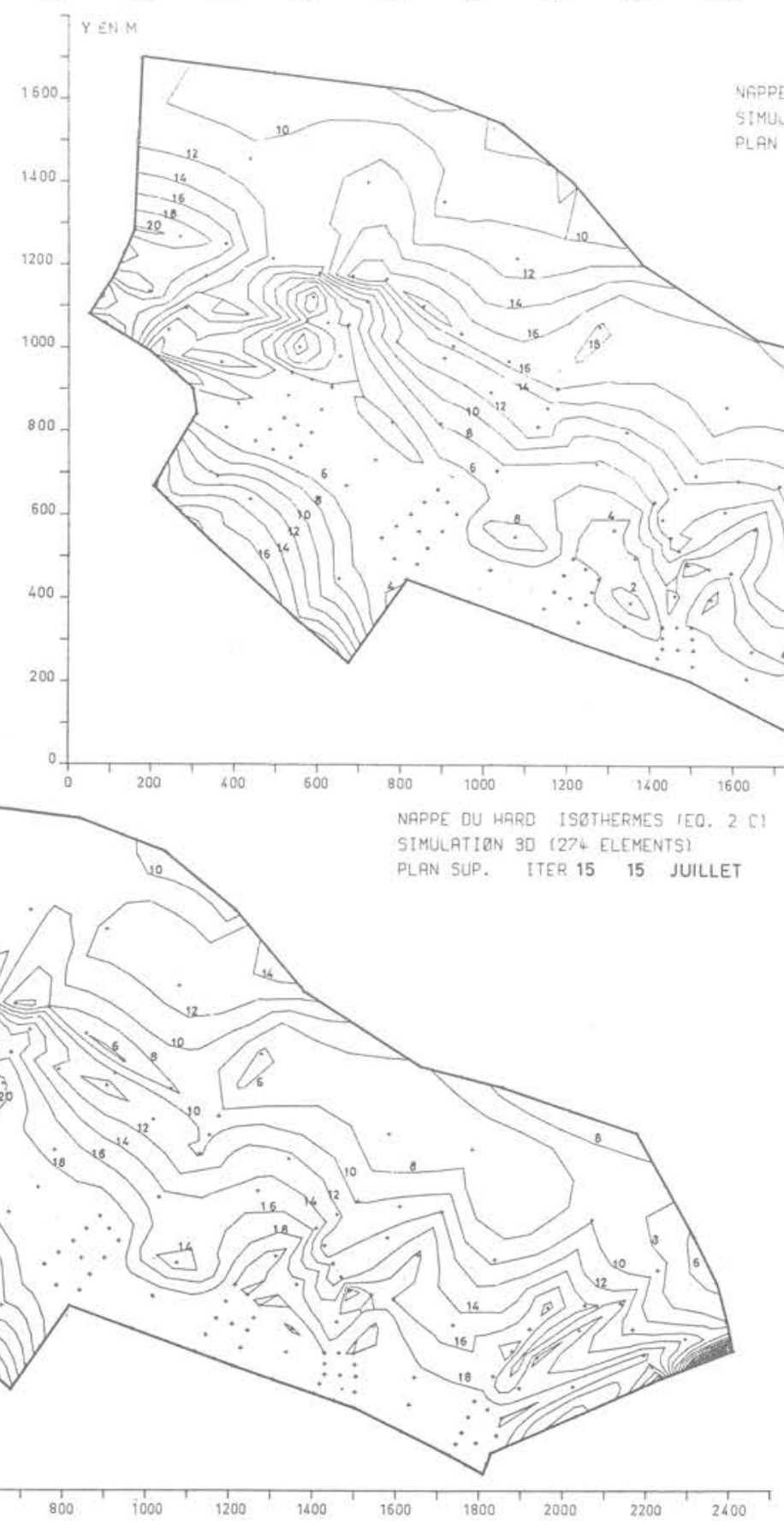

Fig. 18. - Nappe du Hard, isothermes de juillet. 

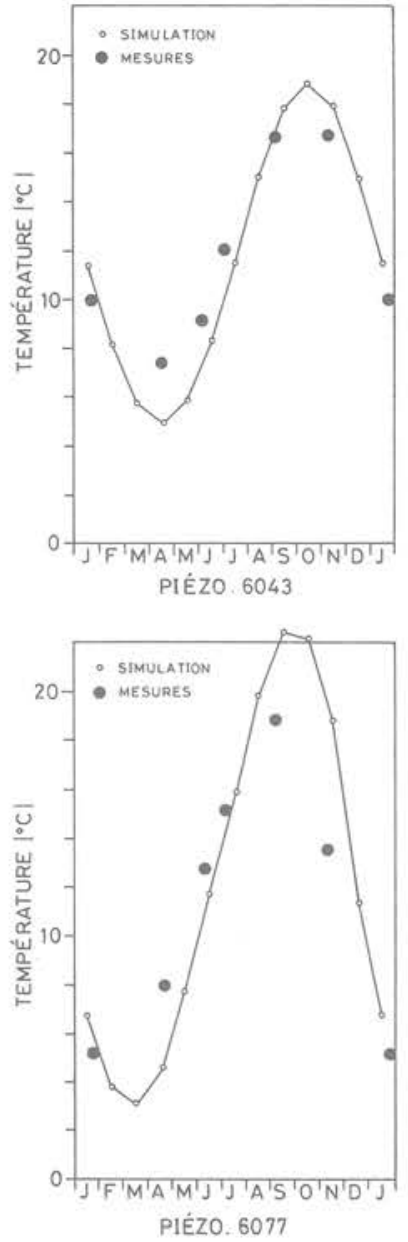
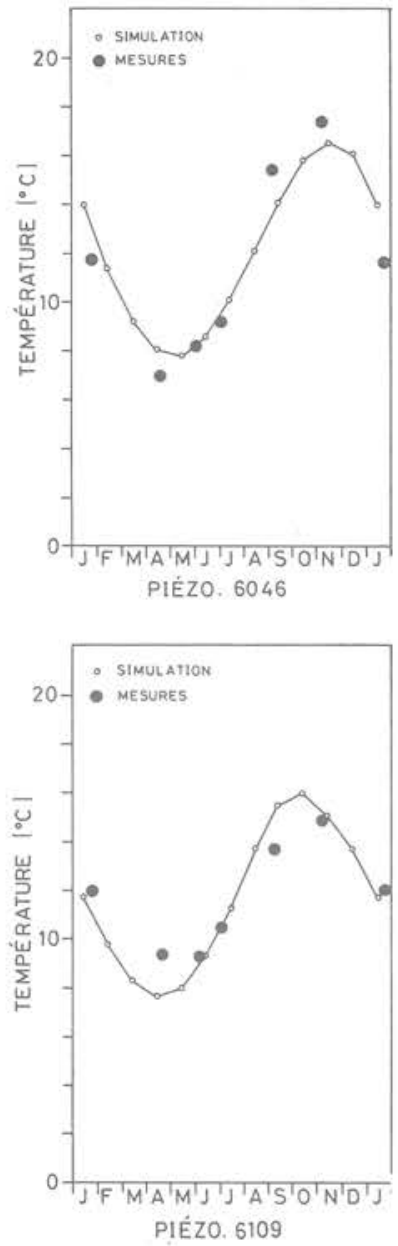

Il faut donc, avant tout, bien définir les caractéristiques hydrauliques. Si l'on dispose de mesures piézométriques en nombre suffisant, il est possible d'évaluer avec une bonne précision le coefficient de perméabilité de Darcy. Mais les erreurs les plus grandes sont dues au choix souvent assez arbitraire des conditions aux limites, car les données géologiques sont en général insuffisamment précises.

\section{ESSAIS DE CHARGE AVEC PLAQUES RIGIDES CIRCULAIRES}

(voir publication [6])

Les essais de charge sur plaque circulaire rigide sont normalisés en Suisse comme essais de contrôle de la qualité du compactage des graves de fondations routières (fig. 20). Le résultat de l'essai se traduit par ce que nous appelons le module de compressibilité $\mathrm{M}_{\mathrm{E}}$ qui est calculé par la relation :

où :

$$
\mathrm{M}_{\mathrm{E}}=\frac{\Delta \sigma}{z_{o}} 2 \mathrm{R}
$$

$\Delta \sigma$; variation de la pression moyenne sous la plaque, en $\mathrm{kPa}$, provoquant un déplacement vertical $z_{o}$ en $\mathrm{cm}$;

$2 \mathrm{R}$ : diamètre de la plaque.

Il s'agit d'un pseudo-module d'élasticité, puisque dans le cas d'une telle plaque, la théorie de l'élasticité donne pour le module d'élasticité : $\mathrm{E}=\frac{\Delta \sigma}{z_{o}} \frac{\pi}{2} \mathrm{R}\left(1-\mu^{2}\right)$, où $\mu$ est le module de Poisson.

Pour une valeur donnée de $\Delta \sigma$, de $\mathrm{R}$ et de $\mu$, le module $\mathrm{E}$ ne dépend que du tassement de la plaque $z_{o}$. Le critère de qualité du compactage est donc simplement le tassement moyen de la plaque.

L'interprétation des résultats de ce type d'essais est parfois difficile et discutable; il est influencé, non
Fig. 19. - Comparaison des températures moyennes calculées et mesurées dans quatre piézomètres.

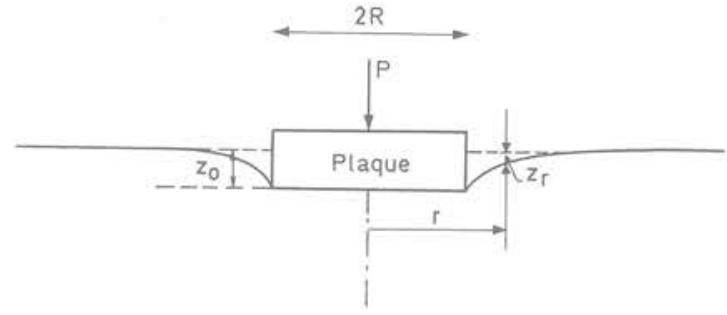

Fig. 20. - Plaque de charge circulaire rigide.

seulement par la compacité de la grave, mais aussi par sa teneur en eau et par les conditions d'appui de la plaque. C'est pourquoi nous avons eu l'idée d'améliorer le critère de qualité en utilisant une autre information de l'essai, la déformation de la surface du sol autour de la plaque de charge.

Il y a quelques années, des essais de charge systématiques ont été exécutés dans ce but sur une couche de grave. La déformée de la surface du sol en dehors de la plaque a été mesurée, selon trois rayons, le long de chacun desquels étaient placés trois comparateurs. 
Les essais ont été répétés de nombreuses fois pour quatre degrés de compactage de la grave. En tout, cent douze essais de charge ont été exécutés pour diverses valeurs de la pression moyenne sous la plaque, comprises entre 50 et $450 \mathrm{kPa}(0.5$ à 4.5 bars $)$.

La mesure de la déformée en dehors de la plaque permet de constater l'écart qui existe entre les résultats expérimentaux et la déformée calculée par la théorie de l'élasticité.

Rappelons quelques résultats de la théorie de l'élasticité appliquée au cas de la plaque rigide circulaire.

La déformée de la surface du sol en dehors de la plaque est donnée par le déplacement vertical $z_{r}$ :

$$
z_{r}=\Delta \sigma \cdot R \frac{1-\mu^{2}}{E} \arcsin \mathrm{R} / \mathrm{r}
$$

$r$ étant la distance du point considéré au centre de la plaque.

Le déplacement vertical de la plaque, $z_{o}$, est obtenu pour $r=\mathrm{R}$; d'où :

$$
z_{o}=\Delta \sigma \cdot \mathrm{R} \frac{1-\mu^{2}}{\mathrm{E}} \cdot \frac{\pi}{2} \text {. }
$$

La contrainte de contact en tout point sous la plaque $\sigma_{z_{r}}$ est donnée par (fig. 21) :

$$
\sigma_{z_{r}}=\frac{\sigma_{m}}{2} \frac{1}{\sqrt{1-(r / \mathrm{R})^{2}}}
$$

dans laquelle $\sigma_{m}$ est la contrainte moyenne, $\sigma_{m}=\frac{\mathrm{P}}{\pi \mathrm{R}^{2}}$, pour une charge $\mathrm{P}$ sur la plaque

Cette équation montre immédiatement que

$$
\begin{array}{lll}
\text { pour } r=0 & \sigma_{z_{r}}=\sigma_{m} / 2 & \\
\text { pour } r=\sqrt{3 / 2} \mathrm{R} & \sigma_{z_{r}}=\sigma_{m} & (r=0.866 \mathrm{R}) \\
\text { pour } r=\mathrm{R} & \sigma_{z_{r}}=\infty &
\end{array}
$$

On trouve, par ailleurs, facilement que l'aire comprise dans le cercle intérieur de rayon $\sqrt{3 / 2} \mathrm{R}$ est $\mathrm{S}_{i}=\frac{3}{4} \pi \mathrm{R}^{2}$, donc l'anneau compris entre $\sqrt{3} / 2 \mathrm{R}$ et $\mathrm{R}$ a une surface égale au quart de la surface de la plaque entière $S_{e}=\frac{\pi R^{2}}{4}$. De plus, par intégration du diagramme des contraintes de contact, on montre que la part de la charge totale $\mathrm{P}$ reprise sur le cercle intérieur est égale à $\frac{\mathrm{P}}{2}$. On a par conséquent les contraintes moyennes suivantes :

$$
\begin{aligned}
& \text { sur le cercle intérieur } \sigma_{i \text { moy }}=\frac{2}{3} \sigma_{m} \\
& \text { sur l'anneau extérieur } \sigma_{\text {e moy }}=2 \sigma_{m}
\end{aligned}
$$

Pour une plaque de $30 \mathrm{~cm}$ de diamètre, telle que celle des normes suisses, l'anneau extérieur n'a qu'une largeur de $2 \mathrm{~cm}$. C'est donc dans cette bande très étroite que, selon la théorie élastique, les contraintes élevées se concentrent $\sigma_{z_{r}}>\sigma_{m}$, alors que dans la partie centrale $\sigma_{z_{r}}<\sigma_{m}$. Les désordres dans le sol sous la plaque doivent donc être concentrés sous cet anneau extérieur ; c'est là que les déviateurs deviennent grands et que le comportement du matériau n'est plus élastique.

Des expériences nombreuses ont permis de contrôler que les contraintes réelles diffèrent de celles que donne la théorie de l'élasticité, par décharge de la zone annulaire extérieure et légère surcharge de la zone centrale. Citons par exemple les recherches de A.R.
THEORIE ELASTIQUE
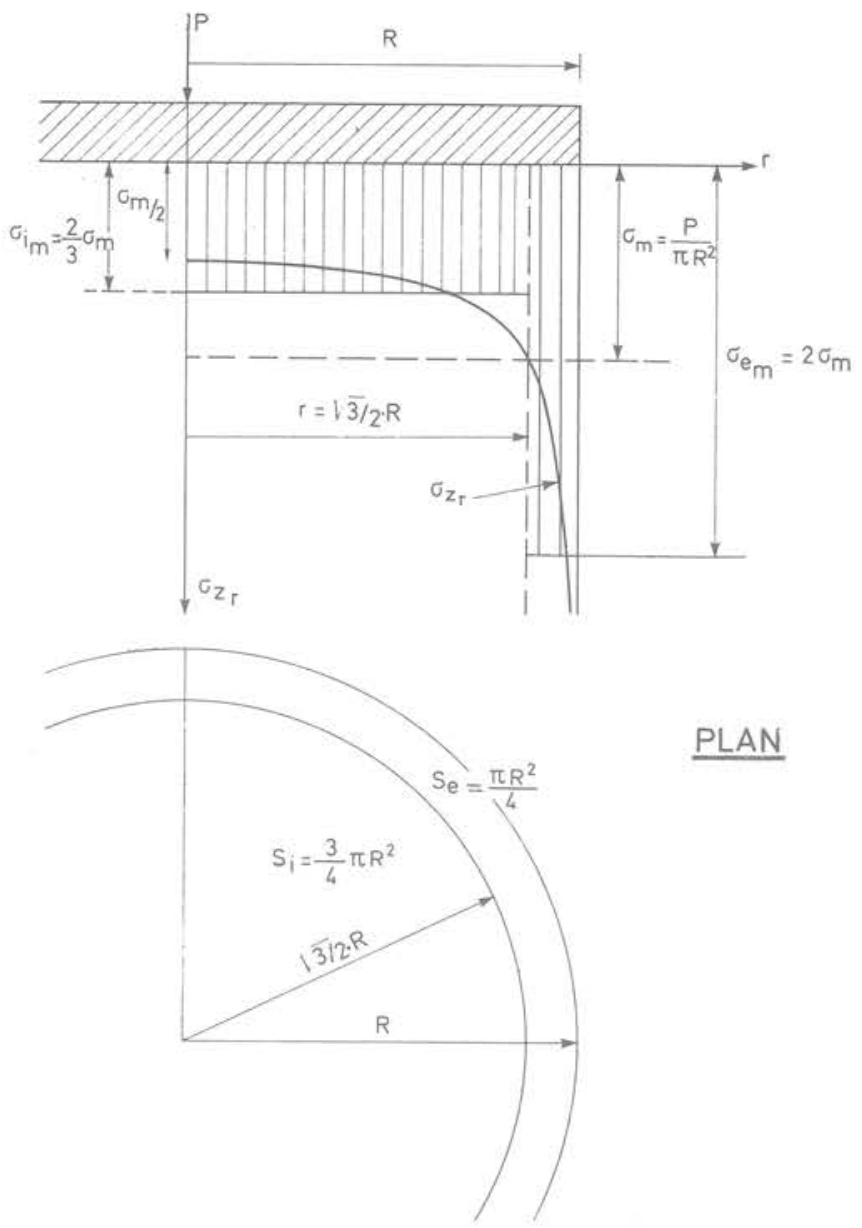

PLAN

Fig. 21. - Diagramme des contraintes de contact sous une plaque circulaire rigide, selon la théorie de l'élasticité.

Wibel de l'Université Fridericiana de Karlsruhe [7] qui a comparé les résultats d'un calcul en élasticité linéaire, ainsi que ceux d'un calcul effectué par la méthode des éléments finis en élasticité non linéaire, à des résultats de mesure de contraintes.

Sous une plaque de charge, le sol est donc refoulé vers l'extérieur et, tout près de la plaque, la surface du sol tasse moins que ne le prévoit la théorie de l'élasticité.

Les mesures de nos expériences confirment clairement ce fait : les figures 22 à 24 montrent les déformées calculées par la théorie de l'élasticité et les déformées moyennes mesurées au voisinage de la plaque pour une grave pratiquement non compactée (fig. 22), pour un compactage moyen (fig. 23) et pour un fort compactage (fig. 24). Les déformées calculées passent par le point $r=15 \mathrm{~cm}$, bord de la plaque à la profondeur $z_{0}$ mesurée. Elles ont été obtenues en admettant qu'elles devaient passer par ce point, ce qui a permis de calculer le module de Young E à l'aide de la formule donnée plus haut avec $\mu=0.3$.

Selon la théorie de l'élasticité, le rapport $z_{o} / z_{r}$ du tassement moyen de la plaque au tassement d'un point de la surface du sol en dehors de la plaque, à une distance $r>\mathrm{R}$ du centre de la plaque, on trouve :

$$
z_{o} / z_{r}=\frac{\pi}{2 \arcsin \mathrm{R} / r}
$$




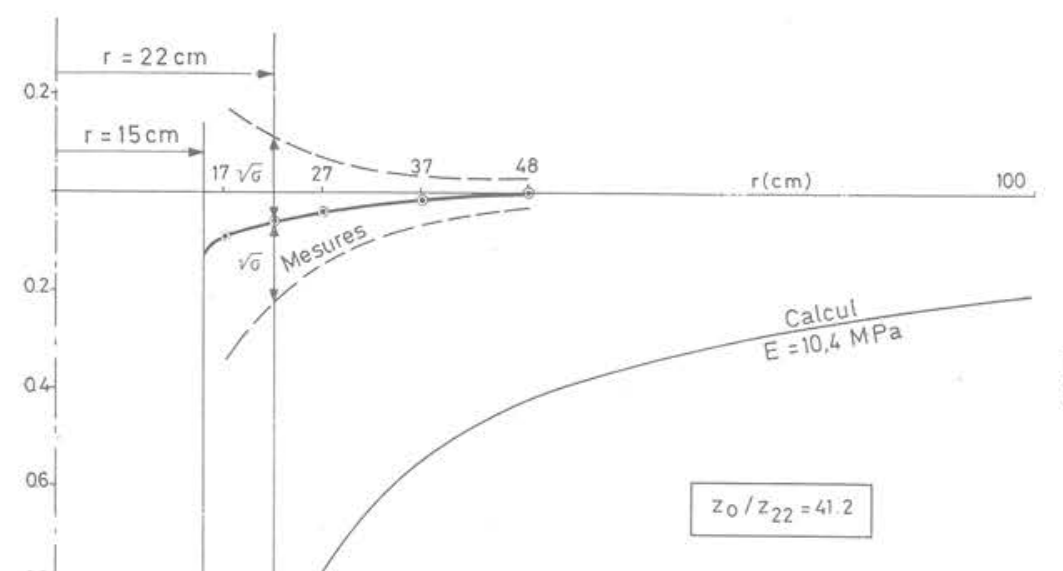

SERIE D'ESSAIS No1-RAPIDES

$$
N=22 \text { essais }
$$

$\Delta \mathrm{P}_{\text {moy }}=50$ à $150 \mathrm{kPa}$

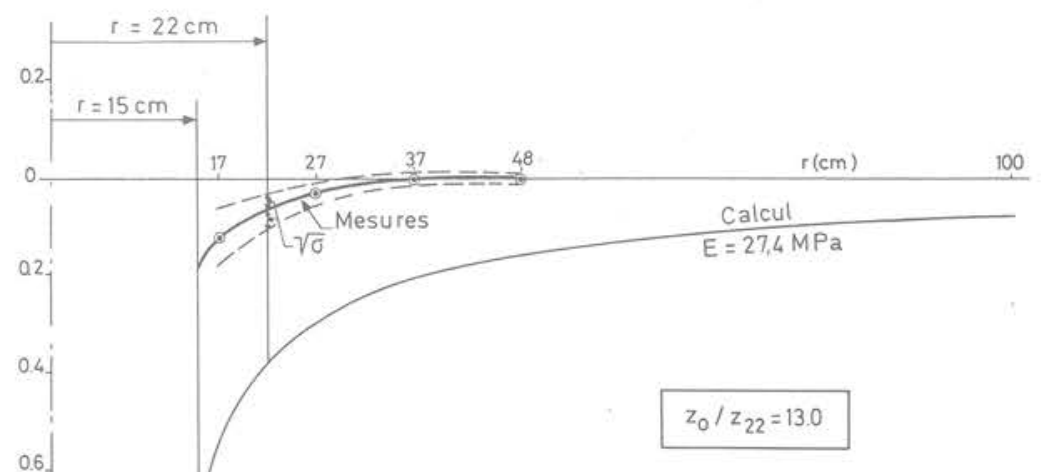

0.6

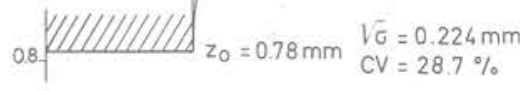

SERIE D'ESSAIS N $N^{\circ} 2$-RAPIDES

$N=20$ essais
$\Delta p_{\text {moy }}=50$ à $150 \mathrm{kPa}$

100

Fig. 24. - Déplacements verticaux calculés et mesurés, fort compactage de la grave.

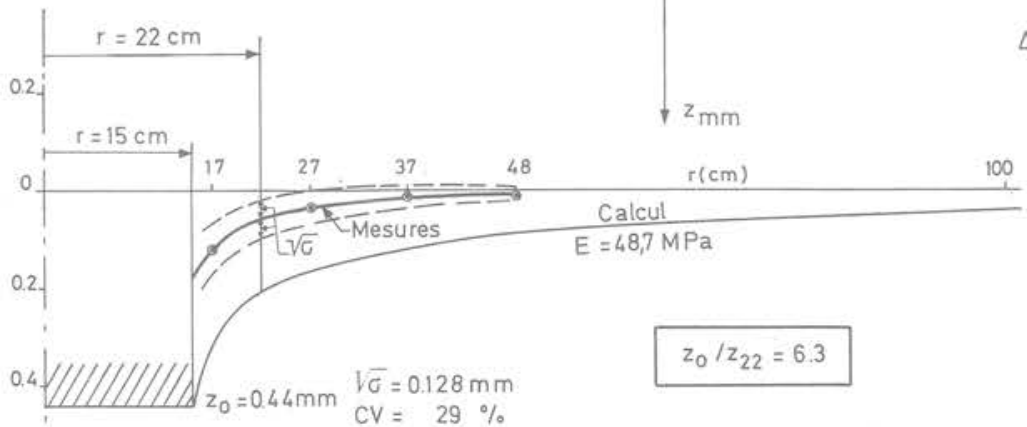

Ce rapport ne dépend ni de la charge appliquée $\mathrm{P}$, ni des caractéristiques élastiques du sol $\mathrm{E}$ et $\mu$. I1 ne dépend que du rapport $\mathrm{R} / r$. Pour une valeur particulière de ce rapport, par exemple $\mathrm{R} / r=15 / 22$, c'està-dire pour les points extérieurs situés à $7 \mathrm{~cm}$ du bord de la plaque de $30 \mathrm{~cm}$ de diamètre, $z_{o} / z_{r}=2.09$.

Si le sol se comportait élastiquement, nous devrions donc trouver toujours cette valeur, quel que soit le module d'élasticité du sol, donc quel que soit son degré 
de compactage. Or, les expériences montrent que ce rapport varie; il est grand pour un sol mal compacté et se rapproche de la valeur de la théorie élastique au fur et à mesure que le compactage est plus intense. Pour les essais effectués, nous avons obtenu les valeurs suivantes :

\begin{tabular}{lrrrr} 
Degré de compactage & & $z_{0} / z_{22}$ & & $\mathrm{E}$ (en $\mathrm{MPa})$ \\
\cline { 1 - 1 } 1. Très faible & & 41.2 & & 10.4 \\
2. moyen & & 13.0 & & 27.4 \\
3. Fort & 6.3 & & 48.7
\end{tabular}

A première vue, ces résultats, qui sont des valeurs moyennes, semblent montrer qu'il n'est pas intéressant d'utiliser de préférence $z_{o} / z_{22}$ comme critère de compactage au lieu du module E. Il n'en est rien, car l'examen détaillé des résultats des essais a montré que ce rapport caractérise mieux le compactage que le module d'élasticité. Il varie plus régulièrement avec le compactage et il est moins influencé par la teneur en eau du sol. Ses valeurs sont moins dispersées.

Il est évident que les très grands écarts constatés entre les déformées calculées (théorie élastique) et mesurées doivent être attribués dans ce cas à l'introduction dans le calcul de mauvaises caractéristiques mécaniques du sol, en particulier les courbes contraintesdéformations réelles ne sont pas linéaires et l'on omet d'introduire les critères de rupture.

Les calculs actuellement en cours, à l'aide de modèles élasto-plastiques, montreront quelles sont les caractéristiques mécaniques qu'il faut introduire pour mieux retrouver les déformées mesurées.

\section{REMBLAIS EXPERIMENTAUX SUR SOLS TRES DEFORMABLES}

(voir publications [8], [9], [10])

La construction du réseau des autoroutes en Suisse a mis les ingénieurs routiers en présence d'un problème discuté, celui de la mise en place de remblais sur sols très déformables.

Dans les plaines et les vallées de Suisse, les couches superficielles sont souvent constituées de tourbe ou de limons calcaires, appelés malencontreusement craies lacustres, dont l'origine est la précipitation des carbonates dans des nappes d'eau douce. Ces limons, de densité très faible, ont une structure lâche et fragile; ils sont thixotropiques.

La construction de remblais de 7 à $8 \mathrm{~m}$ de hauteur sur ces sols est devenue fréquente pour les autoroutes et pour les voies de circulation transversales. La méthode de construction la plus utilisée en Suisse est celle du préchargement. Il s'agit donc de connaître l'évolution des tassements dans le temps et l'évolution des pressions interstitielles pour éviter tous risques d'instabilité durant la construction.
Pour l'étude de ces problèmes, deux remblais d'essais ont été construits, l'un sur les sols tourbeux de la plaine du Rhône, à l'amont du lac Léman et l'autre sur des limons avec craie lacustre et matières organiques dans la plaine de l'Orbe, à l'amont du lac de Neuchâtel.

En ce qui concerne les déformations du sol sous ces remblais, les remarques principales suivantes peuvent être faites :

Dans les deux cas, les tassements de la surface du sol autour du remblai sont extrêmement faibles en regard des tassements sous le remblai.

Pour le remblai sur tourbe, ces tassements n'ont pas dépassé 4 ou $5 \mathrm{~cm}$, alors que sous le remblai luimême, le tassement maximal atteignait $2.50 \mathrm{~m}$.

Pour le remblai sur limon organique (fig. 25), dont la contruction est beaucoup plus récente, des mesures de déplacements horizontaux ont pu être faites à l'aide d'inclinomètres. Ce remblai est construit au bord d'un

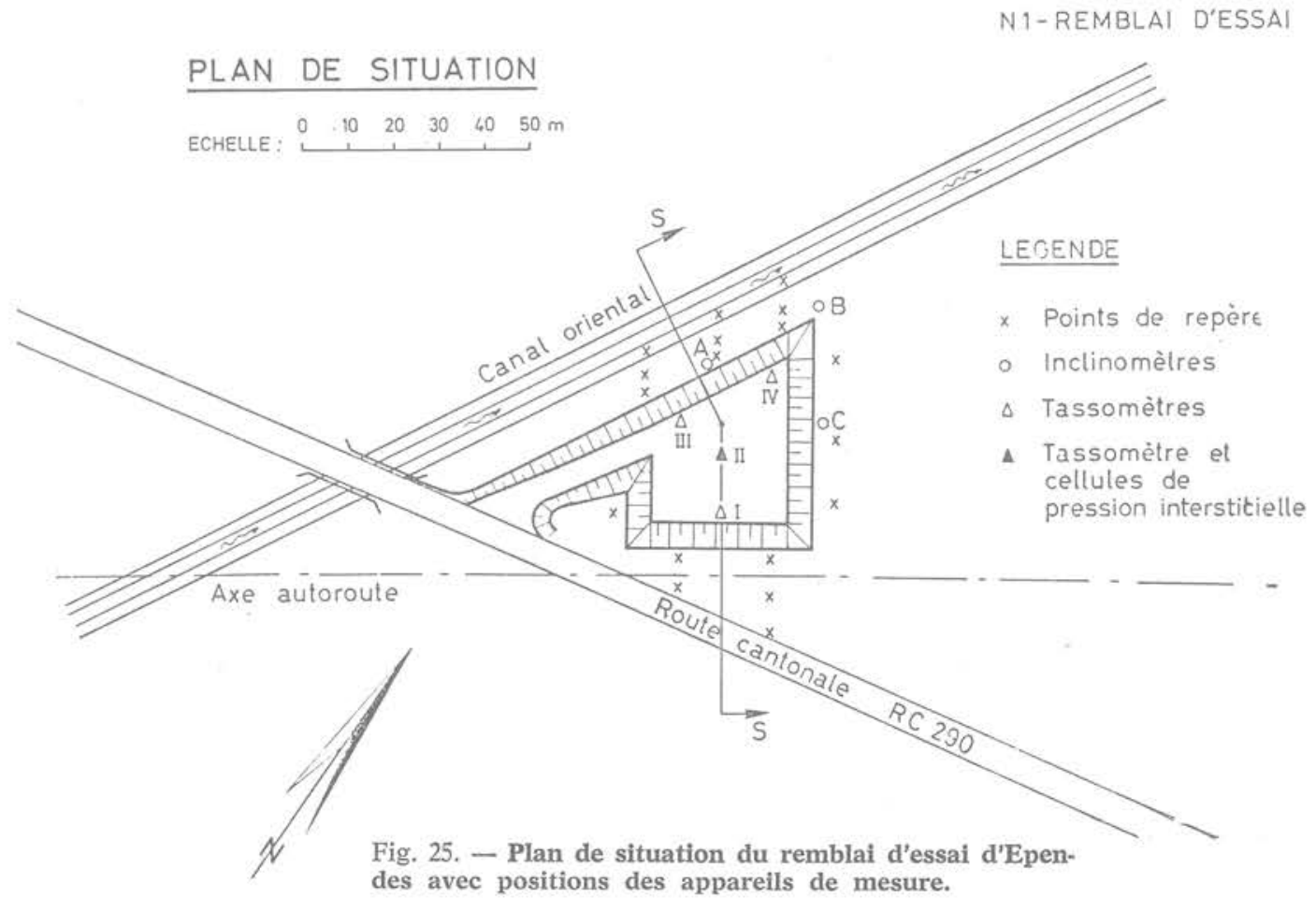


PROFIL EN TRAVERS S-S

ECHEUE $02 \div \quad 6 \quad 8 \quad 10 \mathrm{~m}$

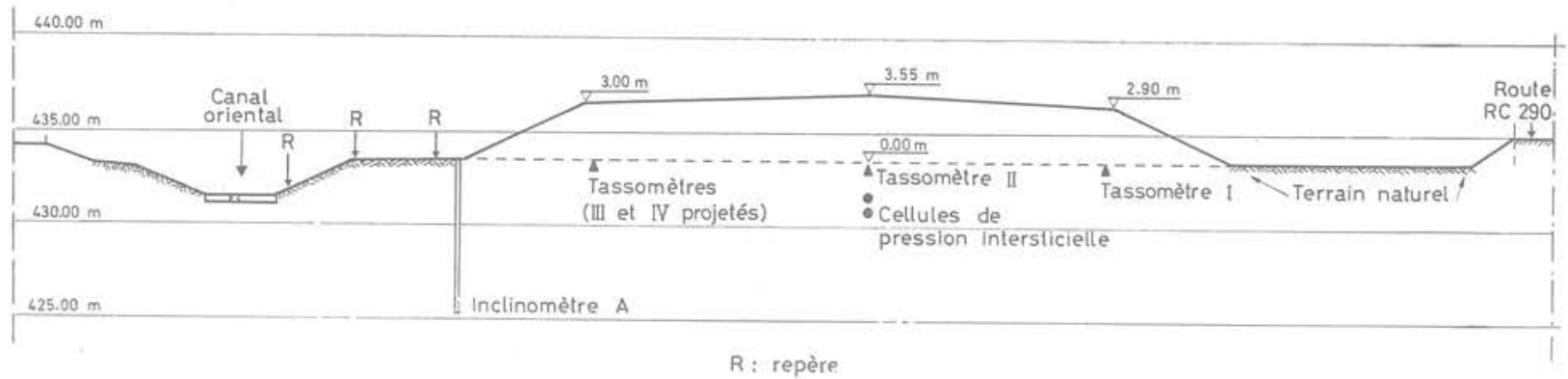

Fig. 26. - Profil du remblai d'essai d'Ependes.

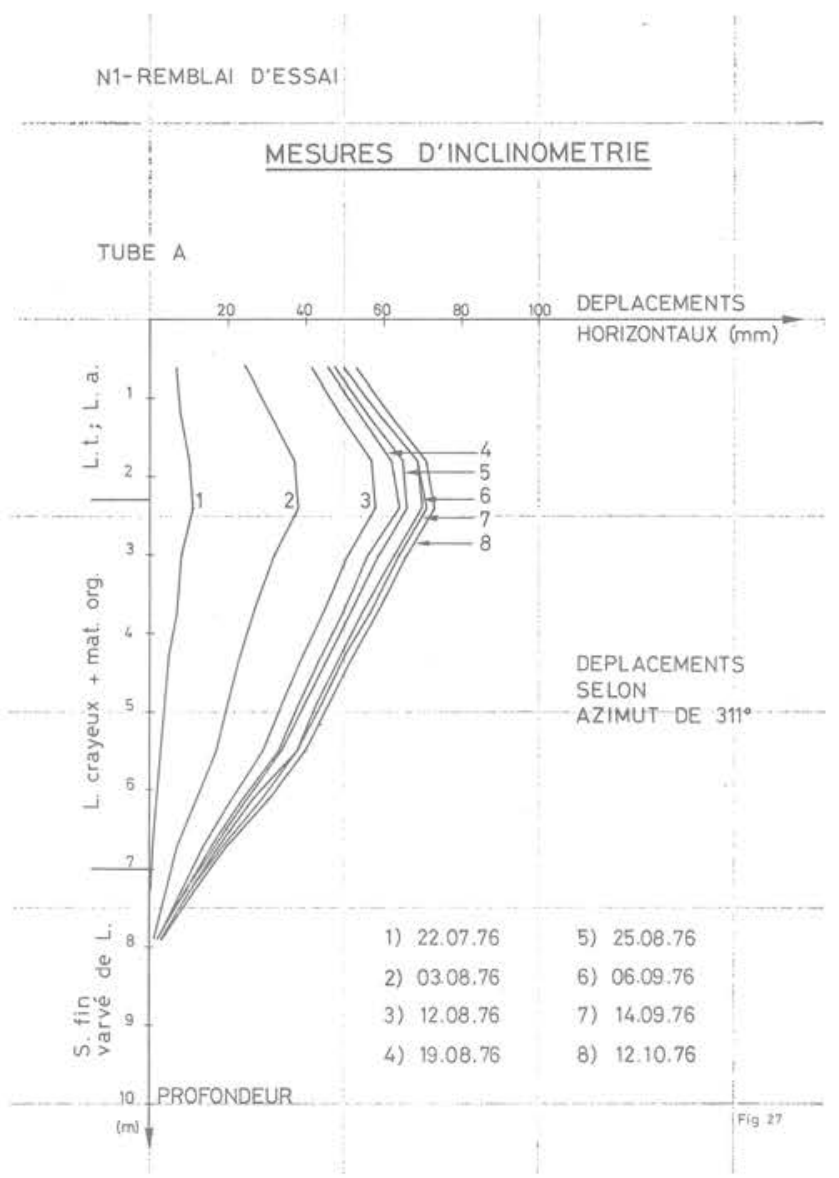

Fig. 27. - Déplacements horizontaux mesurés à l'inclinomètre, côté canal (point A).

petit canal. La figure 26 représente le profil en travers du remblai et indique la position des appareils de mesure.

Dans ce cas également, la surface du sol, même tout près du remblai, n'a pas tassé de plus de 3 ou $4 \mathrm{~cm}$, alors que sous le remblai les tassements maxi-

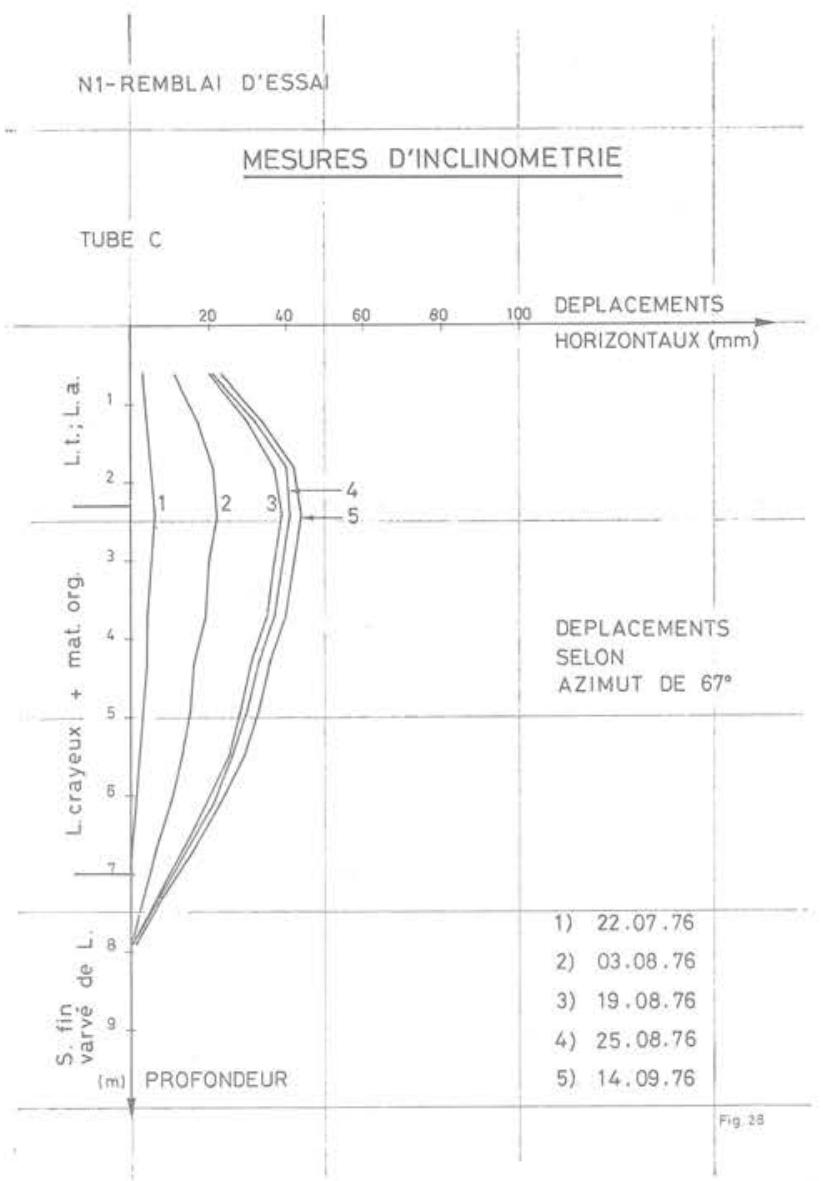

Fig. 28. - Déplacements horizontaux mesurés à l'inclinomètre (point $\mathrm{C}$ ).

maux atteignent environ $60 \mathrm{~cm}$ au centre et $40 \mathrm{~cm}$ au droit du haut des talus.

Les mesures d'inclinométrie montrent que les déplacements horizontaux maximaux se produisent à $2.50 \mathrm{~m}$ de profondeur environ; ils atteignent $7 \mathrm{~cm}$ côté canal et $5 \mathrm{~cm}$ sur les côtés sans canal (fig. 27 et 28 ). 
Les calculs théoriques dans un tel cas nécessitent des moyens très importants si l'on veut tenir compte du comportement non élastique du sol. Ils sont en cours, mais n'ont pas suffisamment avancé pour être présentés. Remarquons que si nous cherchons à calculer à l'aide d'un modèle mathématique perfectionné les déformations dans un tel cas, c'est par intérêt scientifique. Les remblais d'essai ont en effet donné, par les résultats des mesures, toutes les indications nécessaires à la construction.

Nous remarquerons donc finalement qu'ici, comme pour les essais de charge avec plaques, les écarts entre prévisions et observations sont dus, pour l'essentiel, à la méconnaissance des propriétés de déformabilité du sol, dont l'hétérogénéité très marquée accentue les difficultés de l'analyse mathématique.

\section{CONCLUSIONS}

Où doit être porté l'effort principal dans une étude qui concernerait l'un des problèmes types examinés dans cette étude? Le tableau suivant répond en partie à cette question.

\section{CAUSES DES ECARTS ENTRE CALCULS ET OBSERVATIONS}

$$
\begin{array}{r}
\text { Degré d'importance : } 1: \text { le plus grand } \\
5: \text { le plus faible }
\end{array}
$$

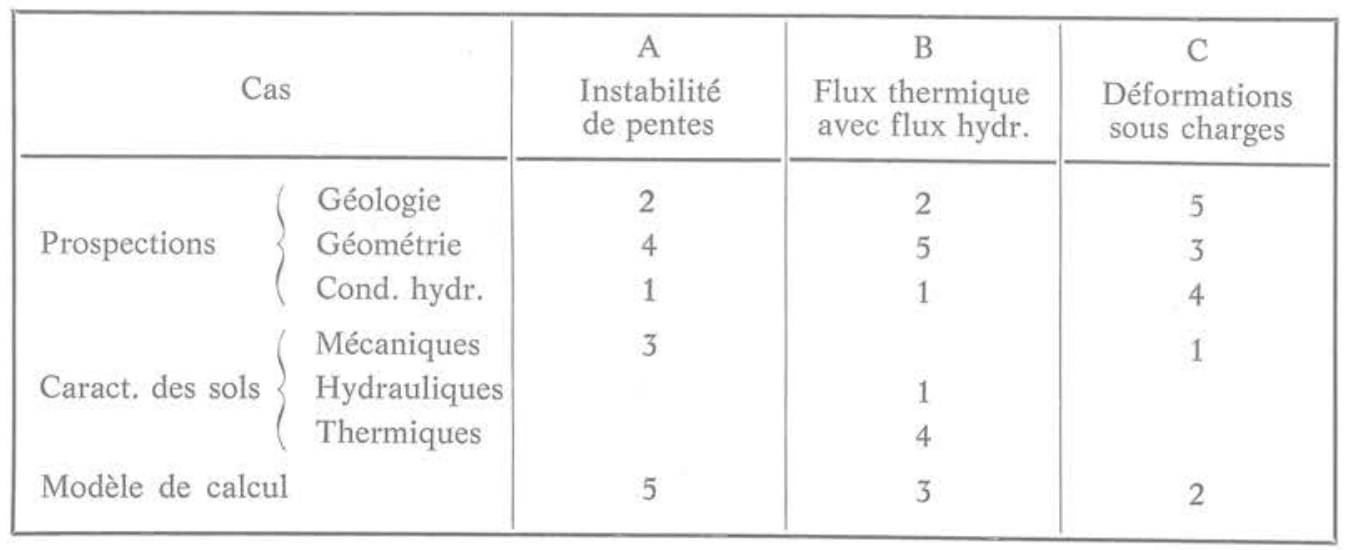

Les cinq études qui ont été décrites appartiennent à trois catégories de cas types.

- instabilités de pentes ;

- flux thermique et hydraulique dans une nappe souterraine ;

. déformations au-dessous d'une surface chargée.

Pour chacun de ces cas types, l'auteur du projet d'un ouvrage devra :

1) recueillir des données sur la géologie, la géométrie et les conditions hydrauliques au moyen de prospections ;

2) recueillir des données sur les caractéristiques des sols par des essais de laboratoire ou in situ;

3) utiliser un modèle de calcul.

Le tableau ci-dessus indique le degré d'importance qu'il faut accorder à chacune de ces phases.

Dans les cas A et B, la priorité est à accorder, à notre avis, aux prospections, puis aux caractéristiques cles sols.

Dans le cas C, ce sont les caractéristiques des sols qui sont causes des erreurs les plus grandes ou l'utilisation d'un mauvais modèle de calcul (élastique par exemple).

Il est bon de se rappeler, lorsqu'on utilise un modèle mathématique basé sur la méthode déterministe, que la dispersion des caractéristiques géotechniques est grande. Généralement, l'hypothèse de base admise est que le sol constitutif d'une couche est homogène. L'expérience permet sans doute de choisir de bonnes valeurs moyennes à partir de quelques essais de laboratoire. Mais on peut se poser la question de savoir si une couche, considérée comme homogène sur la base de sondages normalement espacés, le reste à petite échelle. A cet égard, il est intéressant de savoir quel est le coefficient de variation des caractéristiques géotechniques. A titre d'exemple, je citerai une étude [11] faite sur une couche d'alluvions fines, de $3 \mathrm{~m}$ d'épaisseur, constituée de limon argileux, étudiée à l'aide de six sondages espacés de 50 à $100 \mathrm{~m}$ (fig. 29) dans lesquels vingt-cinq échantillons ont été prélevés dans la couche étudiée. Le coefficient de variation, rapport entre écart type et moyenne, de ces vingt-cinq échantillons est de $1.4 \%$ pour le poids spécifique apparent et $6.3 \%$ pour la teneur en eau. Nous avons voulu vérifier que l'ordre de grandeur de ces coefficients de variation était bien le même pour un volume très petit de cette couche. Seize sondages (fig. 30), distants de $1 \mathrm{~m}$, ont été faits à travers la couche de manière à pouvoir prélever environ cent quatre-vingts échantillons dans un cube de $3 \mathrm{~m}$ d'arête. En groupant ces échantillons de diverses manières et en calculant les coefficients de variation de chaque population d'échantillons, nous avons obtenu les résultats donnés à la figure 31. Elle montre que les coefficients de variation $\mathrm{CV}$ ont sensiblement la même valeur, qu'ils sont en tout cas du même ordre de grandeur, quel que soit le groupement considéré. 


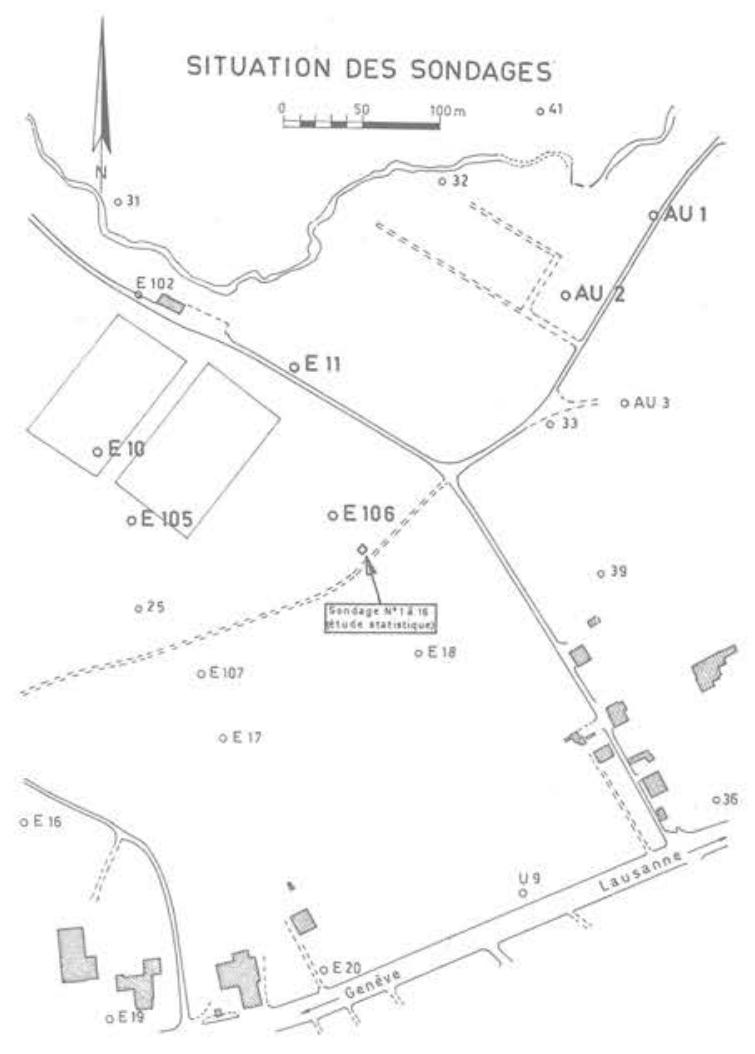

Fig. 29. - Situation des sondages. Site de la nouvelle Ecole Polytechnique Fédérale de Lausanne à Ecublens.

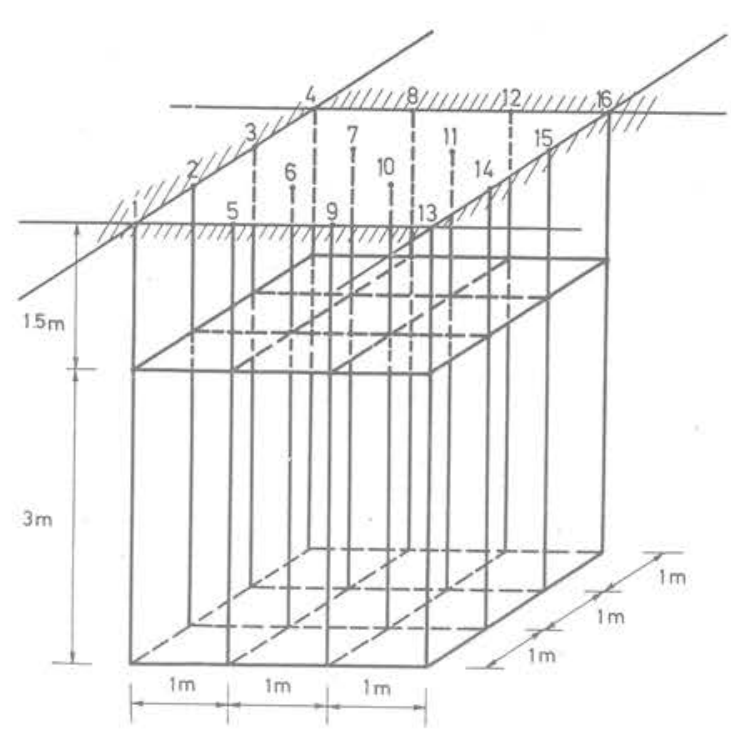

Fig. 30. - Cube de $3 \mathrm{~m} \times 3 \mathrm{~m}$ prospecté à l'aide de six sondages.

Il faut donc craindre, du fait de l'hétérogénéité du sol, de grandes erreurs dans les calculs faisant intervenir la résistance au cisaillement des sols cohérents.

Il en est de même des calculs de déformations. Les essais de charge à la plaque ont montré que la dispersion du tassement de la plaque, pour une même compacité de la grave, correspond à un coefficient de variation de $48 \%$ pour le faible degré de compactage et de l'ordre de $30 \%$ pour la grave compactée.

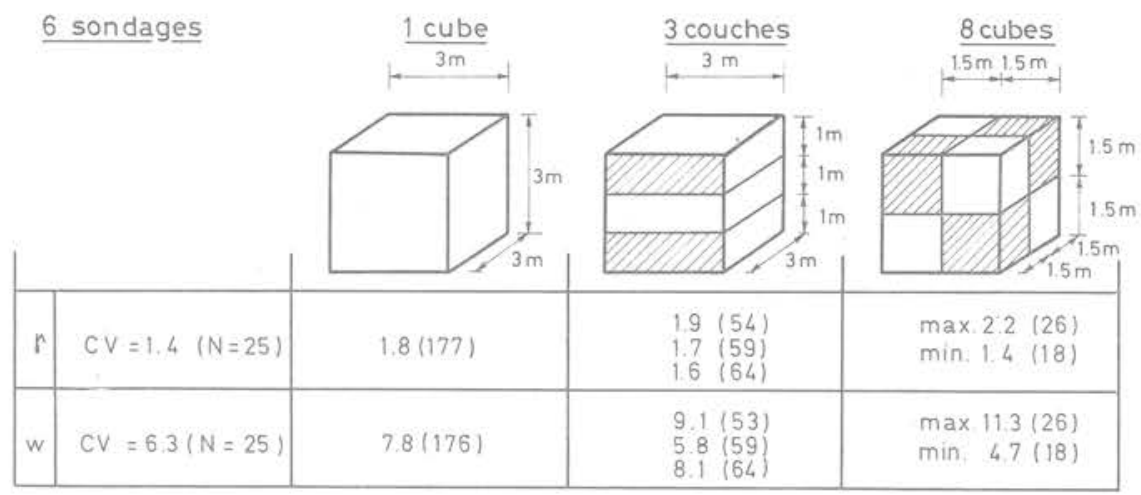

Fig. 31. - Divisions du cube en volumes plus petits et valeurs du coefficient de variation correspondant à chaque ensemble.

$\mathrm{CV}$ : coefficient de variation en $\% ; \mathrm{N}$ : nombre de mesures.

Par contre, si l'on compare les coefficients de variation de diverses caractéristiques géotechniques, on voit que leur ordre de grandeur change énormément d'un cas à l'autre. Les plus grands coefficients de variation sont ceux des propriétés qui font intervenir la cohésion.

$\begin{array}{rrrr} & & \text { CV \% } & \mathrm{N} \\ \gamma: & 1.8 & 177 \\ w: & 7.8 & 176 \\ \Phi: & 13.6 & 32 \\ c: & 114.0 & 32 \\ q_{u}: & 53.7 & 145\end{array}$

L'étude des propriétés des sols est donc utile dans la mesure où elle aide à mieux comprendre qualitativement les phénomènes qui se produisent. Mais dans l'étude d'un ouvrage particulier, il nous paraît plus important de connaître, grâce à des essais simples et nombreux, le domaine de variation d'une caractéristique mécanique, plutôt que de faire une étude très poussée au moyen d'essais de laboratoire très sophistiqués, mais qui ne pourront être faits qu'en petit nombre à cause de leur coût.

Les exemples qui ont été décrits permettent de tirer les conclusions suivantes, valables pour ces types d'études : 
A. En ce qui concerne les problèmes de stabilité de pentes en particulier, les deux causes principales d'écarts entre comportement réel et théorique sont :

1) Les erreurs d'interprétation des prospections qui conduisent à de mauvaises hypothèses sur les conditions hydrauliques.

2) La mauvaise connaissance de la résistance au cisaillement des sols, en particulier de la cohésion.

Il est dans ce cas illusoire d'affiner les méthodes de calcul; celles dont nous disposons sont largement suffisantes. Il faut mettre l'accent sur les prospections et les essais in situ.

B. Dans le cas de l'étude des écoulements souterrains et des températures de nappes en écoulement, des méthodes de calcul très élaborées permettront de faire de bonnes prévisions pour autant que :

- les conditions aux limites de la nappe soient suffisamment bien définies par les prospections et par l'étude géologiques, et que

- le coefficient de perméabilité puisse être contrôlé grâce à des mesures piézométriques.

C. Les calculs de déformations nécessitent la connaissance correcte des courbes contraintes-déformations, qui est essentielle. Les modèles de calcul par éléments finis rendent et rendront de plus en plus de services, car ils permettent de faire intervenir les propriétés plastiques du sol et de mieux traduire les phénomènes dans leur essence. Néanmoins, la précision des valeurs calculées sera d'autant plus faible que le sol sera plus hétérogène.

\section{BIBLIOGRAPHIE}

[1] Encyclopédie illustrée du Pays de Vaud, Vol. 2. Une Terre. Edition Feuille d'avis de Lausanne (1971).

[2] DUBRAY (R.) et FLEISCHER (H.). - " Problèmes posés par la fondation des ponts de l'autoroute du Léman ». Route et Trafic, $\mathrm{n}^{\circ}$ 5/74 (mai 1974).

[3] OTT (J.-C.) et MARGUERAT (J.). - " Le passage inférieur de Saint-Germain à Bussigny ". Route et Trafic, $\mathrm{n}^{\circ}$ 5/74.

[4] HARR (M.-E.). - " Mechanics of particulate media; a probabilistic approach ». En cours de publication chez Mc Graw Hill.

[5] Groupe d'étude de l'Ecole Polytechnique Fédérale de Lausanne et de l'Université de Neuchâtel. " Rapport scientifique et technique sur le comportement des nappes souterraines ». No 4 (novembre 1976).

[6] DYSLI (M.) et RECORDON (E.). - « Déformations superficielles des sols soumis à des essais de charge avec plaques circulaires rigides ".
Rapport de recherche. Laboratoire de géotechnique de l'EPFL (1976).

[7] WIBEL (A.R.). - « Spannungsverteilung unter flachgegründeten Kreisfundamenten auf trockenen Sand ». Universität Fridericiana, Karlsruhe (1971).

[8] RECORDON (E.) et DESPOND (J.-M.). "Remblais sur sols tourbeux ". XIV ${ }^{e}$ Congrès mondial de la route, Prague (1971).

[9] RECORDON (E.). - « Remblais sur sols tourbeux ou craie lacustre ». Bulletin technique de la Suisse romande, $\mathrm{n}^{\circ} 5$ (4 mars 1972)

[10] RECORDON (E.). - " Tassement des sols d'assise sous remblais ". Publication de la Société Suisse de Mécanique des Sols et des Roches, $\mathrm{n}^{\circ} 91$ (mai 1975).

[11] GROLIMUND (J.-P.) et RECORDON (E.). "Etude statistique sur les résultats d'essais de laboratoire effectués sur un sol considéré comme homogène ". Rapport de recherche. Laboratoire de géotechnique de l'EPFL (1972). 

